\title{
Heat Capacities of Fluids: The Performance of Various Equations of State
}

\author{
Zhu, Chenyang; Liu, Xiangyang; He, Maogang; Kontogeorgis, Georgios M.; Liang, Xiaodong
}

Published in:

Journal of Chemical and Engineering Data

Link to article, DOI:

10.1021/acs.jced.0c00649

Publication date:

2020

Document Version

Peer reviewed version

Link back to DTU Orbit

Citation (APA):

Zhu, C., Liu, X., He, M., Kontogeorgis, G. M., \& Liang, X. (2020). Heat Capacities of Fluids: The Performance of Various Equations of State. Journal of Chemical and Engineering Data, 65(12), 5654-5676.

https://doi.org/10.1021/acs.jced.0c00649

\section{General rights}

Copyright and moral rights for the publications made accessible in the public portal are retained by the authors and/or other copyright owners and it is a condition of accessing publications that users recognise and abide by the legal requirements associated with these rights.

- Users may download and print one copy of any publication from the public portal for the purpose of private study or research.

- You may not further distribute the material or use it for any profit-making activity or commercial gain

- You may freely distribute the URL identifying the publication in the public portal 


\section{Heat Capacities of Fluids: The Performance of Various Equations of State}

Chenyang Zhu, ${ }^{\mathrm{a}, \mathrm{b}}$ Xiangyang Liu, ${ }^{\mathrm{b}}$ Maogang He,,${ }^{\mathrm{b}}$ Georgios M. Kontogeorgis, ${ }^{\mathrm{a}}$ and Xiaodong Liang ${ }^{\mathrm{a}, * *}$

${ }^{a}$ Center for Energy Resources Engineering, Department of Chemical and Biochemical Engineering, Technical University of Denmark, DK-2800 Kgs. Lyngby, Denmark

${ }^{b}$ MOE Key Laboratory of Thermo-Fluid Science and Engineering, School of Energy and Power Engineering, Xi'an Jiaotong University, Xi'an 710049, China

*Tel.+862982663863; Fax: +862982663863; Email: mghe@mail.xjtu.edu.cn. **Tel.+4545252877; Fax:+4545882258; Email: xlia@kt.dtu.dk. 
Abstract

11 Heat capacities are fundamental properties of fluids for heat transfer applications.

12 Accurate data can be generally obtained by experimental methods, which are usually

13 expensive, difficult and time-consuming. In terms of the calculations of heat capacities,

14 many models have been proposed in literature. Equations of state represent one of the most

15 promising methods, but their performance has not been systematically studied and

16 extensively reviewed. In this work, calculations and performance of various equations of

17 state for heat capacities are reviewed, and the different contributions to heat capacities are

18 also discussed. The accuracy of the calculated heat capacities, as presented in literature, is

19 also compared for some specific compounds, and the effects of different parametrization

20 strategies as well as association schemes are analyzed. Finally, calculations for both

21 associating and non-associating compounds are performed using two association models,

22 Cubic Plus Association and Perturbed-Chain Statistical Associating Fluid Theory equations

23 of state, for a wide range of compounds for which the heat capacity results from literature

24 are available.

25

\section{Introduction}

27 Heat capacities, including both isochoric and isobaric one, belong to the most important thermophysical properties of fluids. They are related to the temperature derivatives of

29 fundamental thermodynamic functions and they can, therefore, be used to derive other

30 properties such as enthalpy and entropy. ${ }^{1-4}$ In the context of equations of state (EoS), heat 
31 capacities are of interest also for testing the performance limits of such models. ${ }^{5}$ Variations

32 in heat capacities are also required for understanding the changes of the fluid structures ${ }^{1,4}$

33 and heat transfer processes in the industrial aspects. ${ }^{6}$

34 Equations of state are effective tools for representing the thermophysical properties of

35 fluids including heat capacities, which can be calculated by the following two equations:

$$
\begin{gathered}
C_{v}=-T\left(\frac{\partial^{2} F^{\mathrm{res}}}{\partial T^{2}}\right)_{V}+C_{p}^{\mathrm{id}}-R \\
C_{p}=C_{v}-T \frac{(\partial p / \partial T)_{V}^{2}}{(\partial p / \partial V)_{V}}
\end{gathered}
$$

38 where $C_{v}, C_{p}$ and $C_{p}^{\text {id }}$ represent the isochoric, isobaric and ideal gas heat capacities, 39 respectively. $F^{\text {res }}$ is the residual molar Helmholtz free energy, and $R$ is the universal gas 40 constant. At present, EoS based on the perturbation theory are considered among the most 41 popular tools for thermodynamic calculations. Examples of such models are the Statistical 42 Associating Fluid Theory (SAFT) and the Cubic Plus Association (CPA) EoS. The former 43 model was proposed by Chapman et al. ${ }^{7,8}$ Following these original studies, many different 44 EoS based on the SAFT framework were developed..$^{9-15}$ The CPA equation of state (EoS) 45 is a combination of cubic EoS, e.g. Soave-Redlich-Kwong (SRK) and the association 46 contribution from SAFT. It was first developed by Kontogeorgis et al. ${ }^{16}$ in an attempt to 47 balance the accuracy and simplicity of an EoS. ${ }^{17}$ The parameters of SAFT and CPA are 48 obtained from experimental data, most often saturated densities and vapor pressures. 49 Therefore, these models can usually give a satisfactory representation for these two 50 properties, while for some thermodynamic derivative properties e.g. heat capacities, the 
51 performance may get unsatisfactory.

52 In some cases, heat capacities are calculated in order to test the predictive ability of an

53 EoS for second-order thermodynamic derivative properties. For this reason, isochoric $\left(C_{v}\right)$

54 and isobaric $\left(C_{p}\right)$ heat capacities have been calculated by several researchers using different

55 EoS, and the results are summarized in Table 1. Some of the most important studies are

56 discussed hereafter.

57 Palma et al. ${ }^{18-20}$ modified the CPA EoS with a five-parameter function in the attractive 58 term (the so-called $\alpha$ function) and a volume shift, and they analyzed its performance for 59 the estimation of saturated $C_{v}, C_{p}$, and residual $C_{p}\left(C_{p}^{\text {res }}\right)$ for water and alcohols. Then, they 60 compared their results with the original CPA. Polishuk and co-workers ${ }^{15,21-27}$ attached the 61 attractive term of cubic EoS to the SAFT EoS, and they calculated the $C_{v}$ and $C_{p}$ of both 62 associating and non-associating compounds using two SAFT-type models (PC-SAFT, 63 SAFT-VR-Mie) and also, CPA, SBWR and PR for comparison. In addition, the 64 performance of another EoS within the PC-SAFT framework named CP-PC-SAFT for heat 65 capacities of several fluids was also tested by Polishuk and co-workers ${ }^{28-30}$. In their studies, 66 it was mentioned that the available $C_{p}$ data might have noticeable uncertainties, which 67 make the evaluation of the performance of these models difficult. ${ }^{22,30}$ Liang et al., ${ }^{31}$ 68 Diamantonis and Economou, ${ }^{32}$ and de Villiers et al. ${ }^{33}$ compared the accuracy of the PC69 SAFT, SAFT and CPA EoS for the calculation of $C_{v}, C_{p}$, and their residual values. Different 70 parameter sets for these EoS and association schemes of associating compounds were 71 analyzed. In the work of de Villiers et al. ${ }^{33}$, the performance of these models for derivatives 
72 of pressure by volume $(\mathrm{d} p / \mathrm{d} V)$ and temperature $(\mathrm{d} p / \mathrm{d} T)$, which are linked to the isobaric

73 heat capacity, were also discussed. Burgess et al. ${ }^{34}$ tested a new PC-SAFT EoS with a

74 "hybrid" group contribution method by calculating the $C_{p}$ of some non-associating 75 substances. Their calculations were performed at both low-pressure (lower than $7 \mathrm{MPa}$ ) 76 and high-pressure (up to $276 \mathrm{MPa}$ ) conditions. Lafitte et al. ${ }^{35}$ calculated the $C_{p}$ and $C_{p}{ }^{\text {res }}$ of

77 alcohols and their binary mixtures with alkanes through a SAFT-VR EoS based on the Mie 78 potential (SAFT-VR-Mie). The performance of the model for pure alkanes was compared with the SAFT-VR-SW EoS over a wide pressure range. ${ }^{36}$ Following this work, a modified

80 SAFT-VR-Mie with a higher-order perturbation term was then developed by Lafitte et al., ${ }^{37}$

81 and the performance of the new model was tested by several properties including the 82 isobaric heat capacity. The accuracy of calculated $C_{v}$ with SAFT-VR-Mie for the mixtures 83 of fatty acid methyl esters (FAMEs) was analyzed by Perdomo and Gil-Villegas. ${ }^{38}$ A simple 84 mixing rule with a binary interaction parameter fitted to the density and sound velocity data 85 was used in their work. Vega and co-workers ${ }^{39-42}$ estimated the heat capacities of several 86 substances and their mixtures in the subcritical and critical regions using soft-SAFT. A 87 crossover approach on the basis of the renormalization group theory was used in order to 88 improve the performance in the critical region. Maghari et al. ${ }^{43,44}$ investigated the 89 predictive ability of SAFT-BACK for heat capacities and their residual values. In their 90 studies, both alkanes and their mixtures were considered over wide pressure ranges, up to $91300 \mathrm{MPa}$ for alkane mixtures. Another model in the PC-SAFT framework, which is named ePC-SAFT with a Coulomb interaction term for charged species and applicable to ionic 
93 liquids (ILs), was studied by Shen et al. ${ }^{45}$ In their study, the IL molecule is considered as a 94 combination of IL-cation and IL-anion rather than a complete molecule. Except for the 95 CPA- and SAFT-type EoS, there are some other types of EoS being used for heat capacities 96 estimation. Diedrichs et al. ${ }^{46}$ calculated the liquid $C_{p}$ for 33 pure compounds including 97 associating fluids through a group contribution VTPR (Volume Translated Peng-Robinson) 98 EoS, and different parametrization strategies were considered for comparison. Using the 99 PHTC (Perturbed Hard-Trimer-Chain) EoS, Alavianmehr et al. ${ }^{47}$ predicted the $C_{p}$ of 100 several fatty acid ethyl esters (FAEEs) at pressures up to $100 \mathrm{MPa}$, and the results were 101 compared with the calculated data from literature.

102 The equation of state approach is generally considered as an indirect method for heat 103 capacity estimation and can also be used to calculate other required properties of fluids. In 104 addition, methods specifically developed for heat capacity have also been proposed, such 105 as group contribution (GC) methods ${ }^{48-52}$, corresponding states methods ${ }^{52,53}$ and some 106 innovative methods based on quantum mechanics. ${ }^{54-56}$ These methods have been used for 107 the calculations of heat capacities for several compounds, and good agreement between the 108 calculated values and the experimental data was observed. However, the group contribution 109 and corresponding states methods are generally empirical and applicable to the properties 110 at atmospheric pressure and temperatures far from the critical points, while for a global 111 calculation method of heat capacities with a physically sound background, EoS are usually 112 preferred. Therefore, the remainder of this study is focused on EoS, especially those based 113 on the SAFT framework. First, the overall performance of each EoS on heat capacities is 
114 presented according to the literature studies. Then, different equations are compared for 115 some specific compounds containing $n$-alkanes, 1-alochols and water. Next, the 116 performance of the CPA and PC-SAFT EoS is systematically evaluated and discussed via 117 the calculations of the heat capacities of many compounds in the compressed and critical 118 isothermal regions. At last, the manuscript is completed with our conclusions. 
Table 1. Literature studies for the calculation of heat capacities using equations of state

\begin{tabular}{|c|c|c|c|c|c|c|}
\hline Author & Model & $T / \mathrm{K}^{\mathrm{a}}$ & $p / \mathrm{MPa}$ & Substance & Property & Ref. \\
\hline \multirow[t]{3}{*}{ Palma et al. } & Modified CPA & $251-533$ & Saturation & Alcohol & $C_{p}$ & 18 \\
\hline & Modified CPA & $260-576$ & Saturation & Water & $C_{v}, C_{p}$ & 19 \\
\hline & Modified CPA & $260-490$ & Saturation & 1-Alcohol & $C_{v}, C_{p}, C_{p}^{\text {res }}$ & 20 \\
\hline \multirow[t]{17}{*}{ Polishuk and co-workers } & SAFT + Cubic & $150-1400$ & Saturation, & Water, methanol, carbon dioxide, & $C_{v}, C_{p}$ & 15 \\
\hline & SAFT-VR-Mie, PR & & $0.1-1000$ & $n$-pentadecane & & \\
\hline & SAFT + Cubic & $293-413$ & $0.1-160$ & Di-isodecyl Phthalate & $C_{v}$ & 21 \\
\hline & SAFT + Cubic, PC-SAFT & $298-433$ & $0.1-240$ & 1-Alkene & $C_{v}, C_{p}$ & 22 \\
\hline & SAFT + Cubic, PC-SAFT & $80-500$ & Saturation & Halomethane & $C_{v}, C_{p}$ & 23 \\
\hline & SAFT + Cubic, PC-SAFT & $150-420$ & Saturation, & Haloethane & $C_{v}, C_{p}$ & 24 \\
\hline & & & $0.1-120$ & & & \\
\hline & SAFT + Cubic, PC-SAFT & $299-433$ & $0.1-1000$ & $n$-Octane, $n$-octane $+n$-hexadecane & $C_{v}, C_{p}$ & 25 \\
\hline & SAFT + Cubic, SBWR & $243-503$ & Saturation, & $n$-Hexane & $C_{v}$ & 26 \\
\hline & & & $0.1-1000$ & & & \\
\hline & SAFT + Cubic & $298-323$ & $0.1-150$ & Ionic liquid & $C_{v}, C_{p}$ & 27 \\
\hline & PC-SAFT, CPA & & & & & \\
\hline & CP-PC-SAFT & $250-550$ & 0.1 & Ionic liquid & $C_{v}, C_{p}$ & 28 \\
\hline & CP-PC-SAFT, PC-SAFT & $270-1273$ & Saturation, & Water & $C_{v}, C_{p}$ & 29 \\
\hline & & & $30-1000$ & & & \\
\hline & CP-PC-SAFT, & $150-450$ & Saturation & Alcohol, ketone, ether, ester & $C_{p}$ & 30 \\
\hline & GC-PPC-SAFT & & & & & \\
\hline Liang et al. & CPA, PC-SAFT & $280-620$ & Saturation & Water & $C_{v}^{\text {res }}, C_{p}^{\text {res }}$ & 31 \\
\hline Diamantonis and Economou & SAFT, PC-SAFT & $80-695$ & $0.1-20$ & Carbon dioxide, nitrogen, oxygen, & $C_{v}, C_{p}, C_{v}^{\text {res }}$ & 32 \\
\hline & & & & methane, water, hydrogen sulfide & & \\
\hline \multirow[t]{2}{*}{ de Villiers et al. } & CPA, SAFT, PC-SAFT & $0.5-1.1 T_{\mathrm{c}}$ & $0.01-100$ & $n$-Alkane, 1-alcohol & $C_{v}, C_{p}$ & 33 \\
\hline & & $325-570$ & $10-30$ & & & \\
\hline Burgess et al. & GC-PC-SAFT & $323-533$ & $0.1-276$ & Toluene, 2-methylbutane, $n$-decane & $C_{p}$ & 34 \\
\hline
\end{tabular}




\begin{tabular}{|c|c|c|c|c|c|c|}
\hline Author & Model & $T / \mathrm{K}^{\mathrm{a}}$ & $p / \mathrm{MPa}$ & Substance & Property & Ref. \\
\hline \multirow[t]{4}{*}{ Lafitte et al. } & SAFT-VR-Mie & $298-570$ & $0.1-60$ & Alcohol, $n$-alkane +1 -alcohol & $C_{p}, C_{p}^{\text {res }}$ & 35 \\
\hline & SAFT-VR-Mie, & $298-403$ & $0.1-100$ & n-Alkane & $C_{p}, C_{p}^{\text {res }}$ & 36 \\
\hline & SAFT-VR-SW & & & & & \\
\hline & Modified SAFT-VR-Mie & $100-470$ & $0.1-150$ & $n$-Alkane, 1-alcohol, perfluoroalkane & $C_{v}, C_{p}$ & 37 \\
\hline Perdomo and Gil-Villegas & SAFT-VR-SW & $300-700$ & 0.1 & FAME mixture & $C_{v}$ & 38 \\
\hline \multirow[t]{7}{*}{ Vega and co-workers } & soft-SAFT & $293-500$ & $0.1-50$ & $n$-Alkane, perfluoroalkane, & $C_{p}, C_{v}^{\text {res }}, C_{p}^{\text {res }}$ & 39 \\
\hline & & & & 1-alcohol, water & & \\
\hline & (crossover) soft-SAFT & $1.1-1.5 T_{\mathrm{c}}$ & $0.1-60$ & n-Alkane, 1-alcohol & $C_{v}, C_{p}, C_{v}^{\text {res }}, C_{p}^{\text {res }}$ & 40 \\
\hline & & $280-573$ & & & & \\
\hline & (crossover) soft-SAFT & $1.1-1.25 T_{\mathrm{c}}$ & -- & Isobutene, propane + isobutene, & $C_{v}, C_{p}, C_{v}^{\text {res }}, C_{p}^{\text {res }}$ & 41 \\
\hline & & $280-330$ & & $n$-alkane +1 -alcohol & & \\
\hline & (crossover) soft-SAFT & $225-500$ & $0.1-30$ & Perfluoroalkane & $C_{v}, C_{p}, C_{v}^{\text {res }}, C_{p}^{\text {res }}$ & 42 \\
\hline \multirow[t]{2}{*}{ Maghari et al. } & SAFT-BACK & $150-675$ & -- & n-Alkane & $C_{v}, C_{p}, C_{v}^{\text {res }}$ & 43 \\
\hline & SAFT-BACK & $300-400$ & $0.1-300$ & n-Alkane mixture & $C_{v}, C_{p}, C_{v}^{\text {res }}, C_{p}^{\text {res }}$ & 44 \\
\hline Shen et al. & ePC-SAFT & $273-413$ & $0.1-150$ & Ionic liquid & $C_{v}, C_{p}$ & 45 \\
\hline Diedrichs et al. & VTPR & $90-480$ & Saturation & Alkane, ketone, water, etc. & $C_{p}$ & 46 \\
\hline Alavianmehr et al. & PHTC & $286-341$ & $0.1-100$ & FAEE & $C_{p}$ & 47 \\
\hline
\end{tabular}

${ }^{a}$ Temperature and pressure ranges are only for heat capacities. 


\section{Discussion of the Performance of Different Equations of State}

The present study is mainly focused on isochoric and isobaric heat capacities, while the accuracy of other calculated properties, such as vapor-liquid equilibrium (VLE) properties, compressed density, and speed of sound, is also discussed, as ideally we would like to have EoS which can represent a wide range of properties. The performance of various EoS, as presented in literature, for heat capacities and selected additional thermodynamic properties is discussed in this section. The details of calculated results with different models can be found in Table 2, in which the association schemes, the properties used in the parameter estimation methods, and the average absolute relative deviation (AAD), which is defined by the eq 3 , for the various properties and models are also presented.

$$
\operatorname{AAD}(\%)=\frac{1}{N} \sum_{i=1}^{N}\left|\frac{100\left(X_{\mathrm{cal}}-X_{\mathrm{exp}}\right)}{X_{\exp }}\right|
$$

where $X$ is the investigated property, $N$ is the number of data, and subscripts cal and exp represent the calculated and experimental values, respectively.

\subsection{Results with Equations of State}

\subsubsection{CPA EoS}

Palma et al. ${ }^{18-20}$ proposed a modified CPA EoS to calculate the $C_{v}, C_{p}, C_{v}{ }^{\text {res }}$ and $C_{p}{ }^{\text {res }}$ of alcohols and water at saturation. In their works, the original $\alpha$ function (one parameter) was replaced by a 5-parameter function, and two different kinds of ( $T$-independent and $T$ dependent) volume shifts were added to the EoS. The energy parameter and co-volume were obtained by solving the corresponding equations at the critical points in order to 
141 improve the accuracy of the critical properties. Generally, their modified CPA shows

142 satisfactory performance for heat capacities and their residual properties, and the only

143 exception is for the $C_{p}$ of glycerol with an AAD of $15 \% .{ }^{19,20}$ The $T$-independent volume

144 shift used in the modified CPA has no influence on heat capacity calculations, while the $C_{v}$

145 of several alcohols can be better described with a $T$-dependent volume shift.

146 Liang et al. ${ }^{31}$ calculated the $C_{v}{ }^{\text {res }}$ and $C_{p}$ res of water at the saturated condition with CPA,

147 and only the vapor pressure $\left(p_{\text {sat }}\right)$ and saturated liquid density data were used in the

148 parameter estimation procedure. The results show good agreement for $p_{\text {sat }}$ and $\rho_{\text {sat, }, \text {, while }}$

149 the accuracy of $C_{v}{ }^{\text {res }}$ and $C_{p}{ }^{\text {res }}$ at the reduced temperature $\left(T_{\mathrm{r}}\right)$ up to 0.96 is not as good as

$150 p_{\text {sat }}$ and $\rho_{\text {sat,l. }}$ Using the CPA EoS, de Villiers et al. ${ }^{33}$ investigated the heat capacities and

151 speed of sound $(u)$ for $n$-alkanes and 1-alcohols at high pressures. For $n$-alkanes, the AADs

152 of $C_{v}, C_{p}$ and $u$ are lower than $4 \%$ near the critical region $\left(1.06-1.1 T_{\mathrm{c}}\right)$, while the

153 performance on $C_{v}$ and $u$ deteriorates when a lower temperature $\left(0.5-0.9 T_{\mathrm{c}}\right)$ and higher

154 pressure (up to $100 \mathrm{MPa}$ ) region is considered, especially for $u$ with an AAD of $9.3 \%$. Two

155 association schemes (2B and $3 \mathrm{~B}$ ) were used for 1-alcohols by de Villiers et al. ${ }^{33}$ Both

156 schemes, when used in CPA, perform poorly for $u$, with the AAD higher than $12 \%$.

157 Nevertheless, the performance for $C_{p}$ with CPA and both schemes is much better, with the

158 AAD lower than 4.1\%. Considering a $2 \mathrm{~B}$ scheme, Polishuk ${ }^{27}$ tested the performance of

159 CPA for two ionic liquids $\left(\left[\mathrm{C}_{2} \mathrm{mim}\right]\left[\mathrm{NTf}_{2}\right]\right.$ and $\left.\left[\mathrm{C}_{4} \mathrm{mim}\right]\left[\mathrm{NTf}_{2}\right]\right)$. The CPA EoS shows high

160 accuracy of density for these two ILs, while the accuracy of derivative properties for 
$161\left[\mathrm{C}_{4} \mathrm{mim}\right]\left[\mathrm{NTf}_{2}\right]$ is not satisfactory, with the deviations of $16 \%$ and $21 \%$ for $C_{p}$ and $u$, 162 respectively.

163 To sum up these literature studies for CPA, using a more flexible $\alpha$ function with 5 164 parameters, the modified CPA has good performance for heat capacities at the saturated 165 condition, without sacrificing too much the accuracy of other properties. The $T$-dependent 166 volume shift can improve the accuracy of $C_{v}$, but such volume shift may lead to the crossing 167 of isotherms in the $p$ - $V$ plane and negative heat capacities. ${ }^{57}$ Therefore, it is not 168 recommended to use a $T$-dependent volume shift in an EoS for improving its $T$-dependent 169 performance. It should be noticed that, from Table 2, the performance of the original CPA 170 may be only acceptable in some cases, but overall, it predicts rather well the heat capacities 171 of alkanes and alcohols over relatively wide pressure ranges, while the AADs of $u$ are much 172 higher. This may be due to the inadequacy of the model for the description of the $\mathrm{d} p / \mathrm{d} V$.

\subsubsection{Original SAFT EoS}

174 Diamantonis and Economou ${ }^{32}$ studied the performance of original SAFT for heat 175 capacities of some non-associating compounds. The results show satisfactory agreement 176 between the calculated properties for methane, nitrogen, and oxygen, while the accuracy 177 of heat capacities and $u$ for carbon dioxide is barely acceptable. Particularly, the AAD of $178 C_{v}$ for carbon dioxide is $5.9 \%$, while the accuracy of $C_{v}{ }^{\text {res }}$ is much lower with an AAD of $17949 \%$. In the work of Diamantonis and Economou, the heat capacities of hydrogen sulfide and water were also estimated with different association schemes. ${ }^{32}$ For hydrogen sulfide, a one-associating site model (1A) was used to describe the hydrogen bond in $\mathrm{H}_{2} \mathrm{~S}$ dimers. 
The AAD of $C_{v}$ with $1 \mathrm{~A}$ scheme is $4.7 \%$, while the accuracy of $C_{p}$ and $u$ gets worse, with

both AAD higher than $8.5 \%$. Considering hydrogen sulfide as an inert compound (less

parameters than 1A scheme), satisfactory performance for VLE properties, compressed $\rho$,

and $u$ can still be obtained, while the AAD of both heat capacities is higher than $6 \%$. For

water with two association schemes (2B and 4C), neither of them can give good accuracy

187 of second-order derivative properties, especially for the speed of sound, with the AAD of

$188 \quad 19 \%$ using the $2 \mathrm{~B}$ scheme.

189 The heat capacities of $n$-alkanes and 1-alcohols were also estimated by de Villiers et al. ${ }^{33}$

190 using the original SAFT EoS. The results are similar to those with CPA: High accuracy of

$191 n$-alkanes for $C_{v}, C_{p}$ and $u$ is obtained near the critical region, while the AAD of these

192 properties increase when a wider pressure region is considered. The original SAFT coupled

193 with the 2B scheme shows good performance for the $C_{p}$ of 1-alcohols, while the description

194 of $u$ using both 2B and 3B schemes is not satisfactory (deviation around 15\%).

195 In summary, the original SAFT can accurately describe the VLE properties and

196 compressed $\rho$ for both associating and non-associating compounds, but it fails to give a

197 satisfactory description of $u$ for most substances. In addition, the heat capacities of water

198 cannot be well predicted by original SAFT, indicating the need of either a better EoS or an

199 improved parameter estimation method.

$200 \quad$ 2.1.3 PC-SAFT EOS

201 Using the PC-SAFT EoS, Liang et al. ${ }^{31}$ calculated the thermophysical properties of water

202 at $T_{\mathrm{r}}=0.43-0.96$ and vapor pressures. The accuracy of VLE properties is quite satisfactory, 
but the deviation for the residual heat capacities is in all cases higher than $20 \%$. The high

$204 \mathrm{AAD}$ of $C_{p}{ }^{\text {res }}$ and $u$ may be, to some extent, due to the inaccurate description of $\mathrm{d} p / \mathrm{d} V$, 205 which has an AAD of almost 50\%. Polishuk et al. ${ }^{23,24}$ estimated the $C_{p}$ of halomethanes 206 and haloethanes at both saturated and compressed conditions. The AAD of $C_{p}$ are not given 207 in their studies, but we can nevertheless conclude that PC-SAFT can give accurate results 208 for the $C_{p}$ for halomethanes and haloethanes far from the critical points. Using PC-SAFT, 209 Polishuk ${ }^{27}$ calculated the density, heat capacities, and speed of sound for several ILs. The 210 results show good agreement between the calculations and experiments for $\rho$ and $u$, while 211 the AAD of heat capacities can be higher than $43 \%$.

212 Diamantonis and Economou ${ }^{32}$ also calculated the thermophysical properties of some 213 associating and non-associating compounds using PC-SAFT. For non-associating fluids, 214 and for all studied properties, there is very good agreement between the calculated values 215 and experimental data, while the $C_{v}{ }^{\text {res }}$ still has a large AAD of $41 \%$ for carbon dioxide. For 216 hydrogen sulfide with 1A and non-associating schemes, all properties are well estimated 217 by PC-SAFT, no matter whether the association is considered. Although a more rigorous 218 association scheme with four associating sites (4C) is used, the AAD of heat capacities for 219 water are still about $10 \%$, and only the sound velocity data are successfully described by 220 PC-SAFT with 4C scheme among the studied second-order derivative properties of water. 221 However, when the supercritical water over wider temperature (up to $1273 \mathrm{~K}$ ) and pressure 222 (up to $1000 \mathrm{MPa}$ ) ranges is considered, PC-SAFT with 4C scheme provides lower AAD of $223 C_{v}$ and $C_{p}$, which are $6.3 \%$ and $7.1 \%$, respectively, while the deviation of $u$ can increase to 
$2249.0 \% .^{29}$

225 The performance of PC-SAFT for the heat capacities of $n$-alkanes and 1 -alcohols was 226 also tested by de Villiers et al. ${ }^{33}$ The $C_{p}$ of $n$-alkanes is well described by PC-SAFT, 227 although the model shows less accuracy in the vicinity of critical points than over a lower 228 temperature and higher pressure region. Furthermore, the overall performance for $C_{v}$ of $n$ 229 alkanes is satisfactory, while the predicted results of $u$ over a wide temperature and pressure 230 region still need improvement. For 1-alcohols, PC-SAFT with both $2 \mathrm{~B}$ and $3 \mathrm{~B}$ schemes 231 shows high accuracy of $C_{p}$ data but low accuracy for $u$, with the AAD lower than $3 \%$ and 232 higher than $6 \%$, respectively.

233 Except for the original PC-SAFT, some new EoS within the PC-SAFT framework were 234 developed and used to estimate the heat capacities of fluids. By means of the CP-PC-SAFT 235 (Critical Point-based Modified PC-SAFT) and GC-PPC-SAFT (Group Contribution Polar 236 PC-SAFT) EoS, Polishuk and co-workers ${ }^{30}$ calculated the thermophysical properties of 237 alcohols, ketones, ethers, and esters. The former model does not include the association 238 contribution to the residual Helmholtz energy, and the parameters are obtained by solving 239 three equations at the critical points and one equation at the triple point. It is found that CP240 PC-SAFT shows superior performance for compressed $\rho$ and $u$ than GC-PPC-SAFT at 241 pressures up to $861 \mathrm{MPa}$, although the latter one considers both associating and polar 242 interactions. However, CP-PC-SAFT and GC-PPC-SAFT cannot give satisfactory 243 descriptions of $C_{p}$ at saturated conditions. The density and derivative properties of water 244 and ILs were also calculated by Polishuk et al. with CP-PC-SAFT. ${ }^{28,29}$ For water, the model 
shows satisfactory accuracy for density and speed of sound and acceptable performance

246 for heat capacities, while it substantially underestimates these properties at the saturated

247 conditions. $^{29}$ For ionic liquids at the atmospheric pressure, CP-PC-SAFT gives higher

248 deviations of heat capacities $(\leq 7.5 \%)$ than speed of sound $(\leq 4.3 \%)$ and density $(\leq 0.3 \%)$.

249 Nevertheless, as concluded by the authors, the model still gives a robust estimation of $C_{p}$

250 data considering their relatively high uncertainty. ${ }^{28}$ Without considering the polar and

251 association terms in GC-PPC-SAFT, Burgess et al. ${ }^{34}$ calculated the $C_{p}$ and $u$ of three non-

252 associating compounds. Both properties are well described by GC-PC-SAFT, especially in

253 the low-pressure region. In order to estimate the thermophysical properties of ILs, Shen et

254 al. $^{45}$ added the Coulomb interaction term to the PC-SAFT EoS. The parameters in their

255 model (the so-called ePC-SAFT) are only correlated by compressed $\rho$ data, since accurate

256 vapor pressure data of ILs are not available. Although better results are obtained by

257 regarding an IL as a combination of IL-cation and IL-anion than treating it as a single

258 molecule, the performance of ePC-SAFT for heat capacities is still poor. The authors

259 concluded that such large deviations could be due to the lack of experimental ideal gas data,

260 which were estimated using a statistical thermodynamic method in their work.

261 In conclusion, it can be seen that in Table 2, with some exceptions, PC-SAFT can

262 generally show high accuracy for heat capacities, and even give quite satisfactory accuracy

263 of $u$ for some compounds including carbon dioxide, nitrogen, oxygen, methane, hydrogen

264 sulfide, ionic liquids, and water (with 4C scheme).

265

2.1.4 SAFT-VR EoS 

called SAFT-VR) has been also applied for the calculation of heat capacities. Lafitte et

al. ${ }^{35,36}$ calculated the heat capacities of alkanes and alcohols using two versions of SAFT-

VR combined with either square well (SAFT-VR-SW) or Mie potentials (SAFT-VR-Mie).

The results of all of the investigated properties with SAFT-VR-Mie show very good

agreement with the experimental data for both 1-alcohols and branched alcohols. The AAD

of $C_{p}$ and $u$ for all alcohols are lower than $1.5 \%$ and $3.0 \%$, respectively, and the AAD of

$273 C_{p}{ }^{\text {res }}$ for methanol in a wide temperature range $(T=300-700 \mathrm{~K})$ and at $50 \mathrm{MPa}$ is only $6.2 \%$.

The performance of SAFT-VR-Mie on VLE properties and compressed $\rho$ for $n$-alkanes is

mostly satisfactory for compounds with the carbon atom numbers less than 22 , while the

AAD of $p_{\text {sat }}$ can reach to $18 \%$ for hexatriacontane. The SAFT-VR-SW EoS even shows

high inaccuracy of $p_{\text {sat }}$ for decane with the AAD of $47 \%$. Both SAFT-VR-Mie and SAFT-

VR-SW cannot give high accuracy of heat capacity for $n$-alkanes, and the AAD of $C_{p}$ and

$279 C_{p}{ }^{\text {res }}$ are within the ranges of $5.4-8.7 \%$ and $23-40 \%$, respectively. A modified SAFT-VR-

280 Mie with a higher-order perturbation term was also proposed and used to calculate the VLE

281 and derivative properties for $n$-alkanes, 1 -alcohols and perfluoroalkanes by Lafitte et al. ${ }^{37}$

282 This new SAFT-VR-Mie shows satisfactory accuracy for most substances in the

283 compressed liquid phase, while the AAD of $C_{p}$ for methanol and $u$ for perfluoromethane

284 are still noticeable (about 7-9\%).

285 The properties of mixtures of $n$-alkane +1 -alcohol were also calculated by Lafitte et al. ${ }^{35}$

286 using the SAFT-VR-Mie EoS at atmospheric pressure. High accuracy is obtained for 
compressed $\rho, C_{p}, C_{p}$ res , and $u$ without any adjustable interaction parameters. Perdomo and

288 Gil-Villegas ${ }^{38}$ estimated the $C_{v}$ and $u$ values of the mixtures of fatty acid methyl esters

289 (FAMEs) at $p=0.1 \mathrm{MPa}$. They have considered association contributions for these

290 compounds in their work. The $C_{v}$ of FAME mixtures are well described by SAFT-VR-SW.

291 However, the accuracy of $u$ is still not satisfactory, although the data of this property are

292 included in the objective function for the parameter estimation.

293 In brief, the SAFT-VR-Mie EoS shows a great potential for calculating the heat 294 capacities and $u$ of alcohols and their mixtures with alkanes. For pure $n$-alkanes, the $u$ data 295 can be accurately reproduced by adding them to the parametrization process, while the 296 accuracy of $C_{p}$ still needs improvement. This inaccuracy may be alleviated by including $C_{p}$ 297 data in the parameter estimation procedure or using a higher-order perturbation term.

\subsection{5 soft-SAFT EoS}

The soft-SAFT EoS is a variant of SAFT EoS with the reference term of a Lennard-Jones

300 (LJ) fluid, which includes both the repulsive and attractive interactions for chains formation.

301 By means of the soft-SAFT EoS, Vega and co-workers ${ }^{39}$ investigated the heat capacities and their residual values for both associating and non-associating fluids at high pressures.

303 For non-associating compounds ( $n$-alkanes and perfluoroalkanes), in most cases, it seems 304 sufficient to obtain good results of VLE properties and heat capacities at $T_{\mathrm{r}}=0.7-0.9$ and 305 pressure up to $30 \mathrm{MPa}$ only to use the VLE data in the parameter estimation. For the associating substances of 1-achohols and water, the $C_{p}$ data should be added to the parameter estimation procedure for obtaining better results of heat capacities. For example, 
the AAD of $C_{p}$ for 1 -hexanol can reach to $13 \%$ without correlating the parameters to $C_{p}$

309 data, while such deviation can be reduced to $5.4 \%$ when the parameters are refitted to both

310 VLE properties and $C_{p}$. However, although soft-SAFT can give high accuracy of calculated

311 heat capacities, the description of the $C_{v}{ }^{\text {res }}$ and the $C_{p}{ }^{\text {res }}$ near the critical points is still poor.

312 A crossover soft-SAFT EoS with a renormalization group (RG) method was also

313 developed by Vega and co-workers, ${ }^{40-42}$ and the model showed rather good accuracy of

314 VLE properties for $n$-alkanes, isobutene and perfluoroalkanes. In addition, satisfactory

315 performance for heat capacities for perfluoroethane is observed at the gas and liquid

316 subcritical conditions $\left(T_{\mathrm{r}}=0.77-0.94\right.$ and $\left.p=0.1-30 \mathrm{MPa}\right)$, with the AAD about $5 \%$ for both

$317 C_{v}$ and $C_{p}$. The crossover soft-SAFT EoS coupled with mixing rules which include two

318 binary parameters, was also applied to the calculations of heat capacities for propane +

319 isobutene and $n$-alkane +1 -alcohol. ${ }^{41}$ It can be observed that both $C_{v}$ and $C_{p}$ are precisely

320 represented at atmospheric pressure.

321 Considering the high accuracy for $C_{p}{ }^{\text {id }}$, the soft-SAFT EoS can be regarded as a good

322 tool for describing the heat capacities for non-associating compounds. As for associating

323 fluids, one of the prerequisites for getting acceptable accuracy of heat capacity is

324 correlating the adjustable parameters to $C_{p}$ data, although this is not necessary for non-

325 associating compounds like $n$-alkanes. Furthermore, for the residual heat capacities, the

326 calculated results with soft-SAFT show significant deviations from the experiments,

327 especially for the values in the vicinity of the critical points.

$328 \quad$ 2.1.6 Other $\operatorname{EoS}$ 
To avoid the numerical pitfalls of the EoS and give a better description on critical data,

330 Polishuk and co-workers ${ }^{15,21-26}$ added an attractive term of cubic EoS to SAFT and

331 developed a new equation of state named SAFT + Cubic. Using the proposed model, they

332 calculated the thermophysical properties of several fluids at both saturated and compressed

333 conditions, but the values of AAD were not given. Generally, as shown in their works, the

334 SAFT + Cubic EoS describes better compressed $\rho, k_{T}, C_{v}$ and $u$ than PC-SAFT and better

335 compressed $\rho$ and $u$ than SAFT-VR-Mie, while its performance for saturated $C_{v}$ of $n$ -

336 hexane is worse than the SBWR (Soave-Benedict-Webb-Rubin) EoS. In addition,

337 although the results with SAFT + Cubic are better than PC-SAFT and SAFT-VR-Mie, it

338 still shows large deviations for $C_{v}$, and its performance on heat capacity deteriorates with

339 the increase of $\rho .{ }^{15}$ Nevertheless, it should be emphasized again that, as stated by the

340 authors, the used $C_{p}$ data may have significant uncertainties, thus a precise comparison of

341 models may be difficult. ${ }^{22,30}$ In order to describe the properties of ionic liquids, a

342 generalized version of SAFT + Cubic were developed and used by Polishuk. ${ }^{27}$ With the

343 parameters only obtained by two density data, very impressive performance of the model

344 can be observed, and the values of AAD for all investigated properties are lower than $3.1 \%$.

345 In conclusion, the performance of SAFT + Cubic for heat capacities requires further study

346 as well as the effects of different parametrization methods with this model.

347 Combining the BACK and SAFT EoS, the performance of the so-called SAFT-BACK

348 on second-order derivative properties was tested by Maghari et al. ${ }^{43,44}$ The results for $C_{v}$,

$349 C_{p}, C_{v}{ }^{\text {res }}, C_{p}{ }^{\text {res }}$, and $u$ for $n$-alkanes and their mixtures indicate good agreement with 
experimental data, and no interaction parameter is used for mixtures. Using the volume

351 translated Peng-Robinson (VTPR) EoS, Diedrichs et al. ${ }^{46}$ estimated the saturated properties

352 of some pure compounds including alkanes, ketones, water, etc. In their work, an

353 exponential $\alpha$ function and a $T$-independent volume shift were used, and the parameters of

354 the EoS were correlated to $p_{\text {sat }}$ and $C_{p}$ data. The accuracy of VTPR for $p_{\text {sat, }} \Delta H_{\text {vap }}$ and $C_{p}$ is

355 very good, with the AAD of $0.9 \%, 1.2 \%$, and $0.8 \%$, respectively. This good agreement

356 between the calculated $C_{p}$ and the experimental values can be partially explained by the

357 fact that the parameters of VTPR are correlated to the $C_{p}$ data. However, it should be noted

358 that, in the study of Diedrichs et al, these properties are sometimes calculated over

359 relatively low reduced temperature ranges. For example, the temperatures of water in their

360 work are from 273 to $373 \mathrm{~K}$, corresponding to a reduced temperature range of $0.42-0.58$.

361 Alavianmehr et al. ${ }^{47}$ calculated the properties of fatty acid ethyl esters (FAEEs) with the

362 perturbed hard-trimer-chain (PHTC) EoS, for which the parameters were only fitted to the

363 compressed $\rho$. A satisfactory accuracy of $\rho, C_{p}$, and $u$ can be observed in their study.

364 However, their study is mostly performed over a narrow temperature range, with the

365 conditions of FAEEs commonly far from their critical points. Therefore, a further study at

366 a wider temperature range would be relevant for a more complete evaluation of the

367 performance of the model.

$368 \quad 2.2$ Other Results

369 In many cases, the heat capacities of fluids can be estimated with rather low deviations,

370 but higher deviations can be seen, as expected, when we focus only on the residual values 
371 which are obtained from the thermodynamic models. The overall good results are due to

372 the accurate values of the ideal heat capacity $\left(C_{p}\right.$ id $)$, which is most often the main

373 contribution. ${ }^{18,35,46}$ In order to illustrate the above, we calculated the percentage of $C_{p}$ id $/ C_{p}$

374 for water with the results from the NIST chemistry webbook ${ }^{58}$ at saturated condition and

375 different pressures. The results are shown in Figure 1.

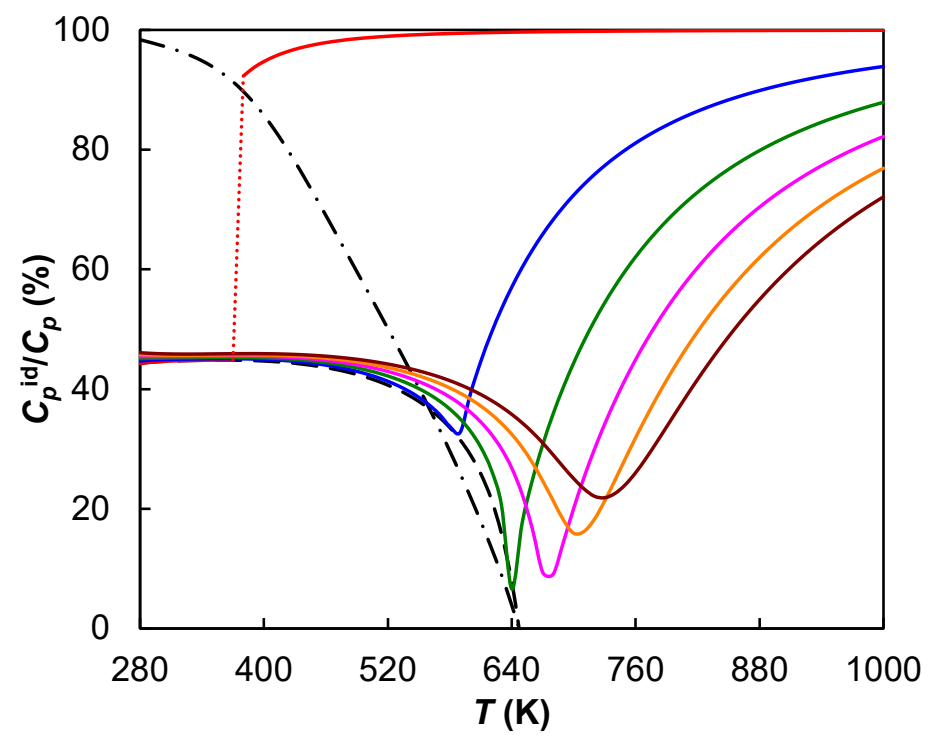

376

377

378

379

380

381

382

Figure 1 . The percentage of ideal gas heat capacity to heat capacity for water with respect to temperature: $(--)$ Saturated liquid, $(-\cdot-)$ Saturated vapor, $(-) 0.1 \mathrm{MPa}(\mathrm{red}),(-) 10$ $\mathrm{MPa}$ (blue), (-) $20 \mathrm{MPa}$ (green), (-) $30 \mathrm{MPa}$ (pink), (-) $40 \mathrm{MPa}$ (orange), (—) $50 \mathrm{MPa}$ (brown).

From Figure 1, It is obvious that, at temperatures much lower than the critical temperature, the proportions of $C_{p}$ id $/ C_{p}$ at different pressures are almost identical, with the value of $44 \%$ or so. This reflects that the pressure can only have a negligible influence on heat capacity in this region. According to Figure 1 and ref. 59, we can know that the liquid 
$385 C_{p}$ at constant pressure and vapor pressure are in close numerical agreement at low reduced 386 temperatures. Therefore, if the liquid saturated $C_{p}$ can be well described using an EoS, the 387 performance for $C_{p}$ at different pressures and low temperatures will also be satisfactory. 388 This can partially explain the reason that the saturated $C_{p}$ data are required in 389 parametrization process in some cases. At temperatures close to the pseudocritical 390 temperatures (at which $C_{p}$ maximum can be obtained along supercritical isobars), the 391 percentages of $C_{p}$ id $/ C_{p}$ decline rapidly to low values. This is the reason that the calculated 392 heat capacities can only achieve quite low accuracy in these regions. Furthermore, with the 393 increase of temperature, the proportions then rise up to higher than $70 \%$ even at high 394 pressures. Obviously, the $C_{p}$ data at higher temperatures are much easier to be well395 described with an equation of state.

396 From the above, we can conclude that the residual contribution is significant in the liquid 397 and near-pseudocritical regions, but at high temperatures, it becomes less important. ${ }^{32}$ For 398 non-associating compounds, the dispersion contribution to the residual heat capacities is 399 dominant. ${ }^{43}$ According to this, the calculation of higher-order perturbation terms in EoS is 400 probably required for a better description of heat capacities. ${ }^{36}$ For associating compounds, 401 the association plays the most important role in the residual heat capacities for relatively 402 short associating chains, such as methanol. ${ }^{40}$ However, with the increase of temperature or 403 molecular length, the contribution of the association term decreases, while the dispersion 404 contribution becomes more significant.

405 Then, as the calculations of speed of sound need both $C_{v}$ and $C_{p}$, we now turn our 
406 attention to the description of $u$. In some cases, such as ePC-SAFT for ILs, ${ }^{45}$ PC-SAFT for 407 water and the original SAFT for hydrogen sulfide, ${ }^{32}$ the AAD of $C_{v}$ and $C_{p}$ are relatively 408 large, while the deviations of $u$ are much lower. The high accuracy of $u$ may be not because 409 of the accurate description of interactions between molecules with the EoS but arise from 410 a compensation of the errors on the $C_{v}$ and $C_{p}$, since the results of $u$ are related to the values 411 of $C_{p} / C_{v}{ }^{24}$ 
Table 2. Summary of the results with different equations of state as presented in literature

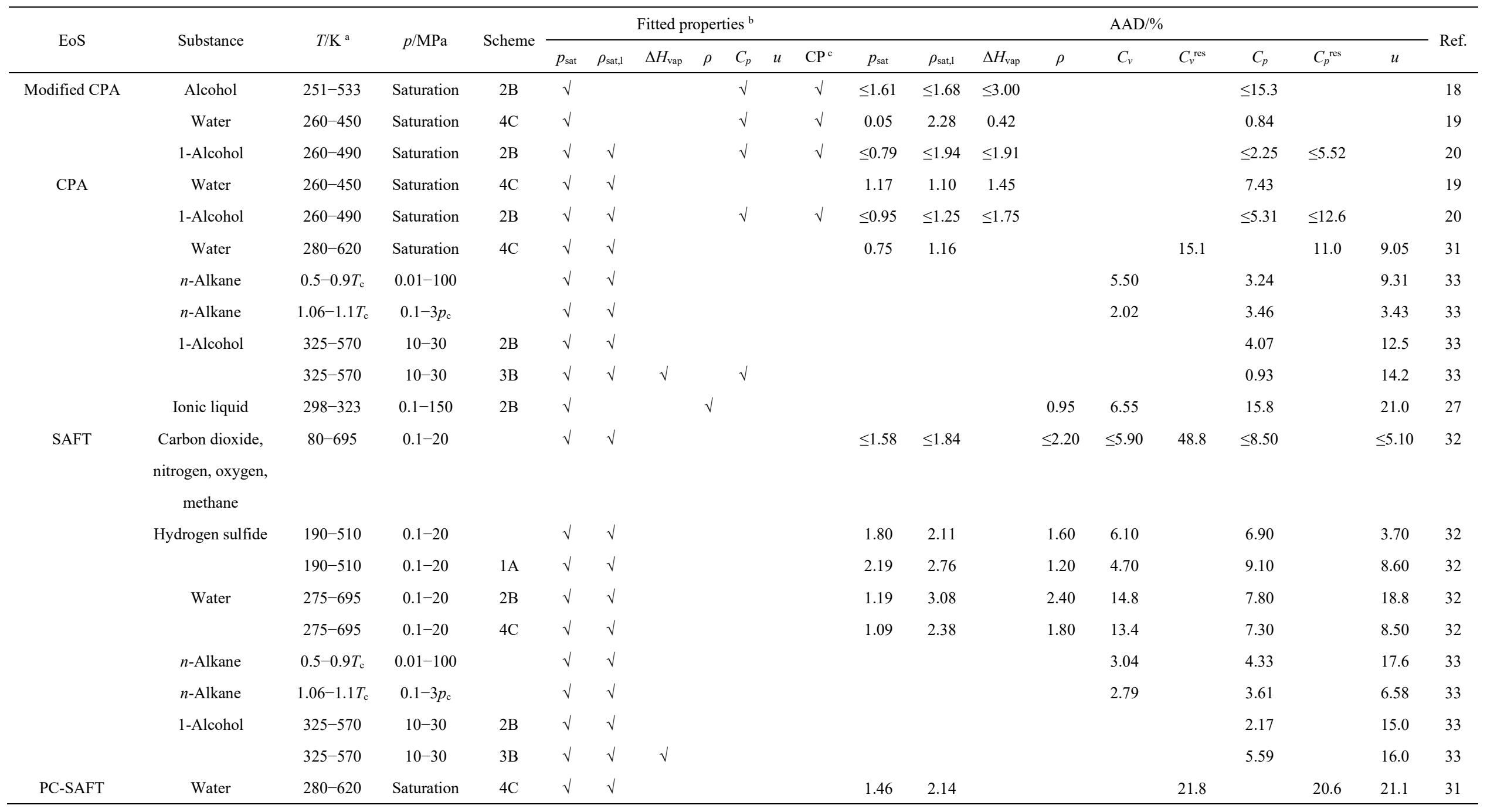




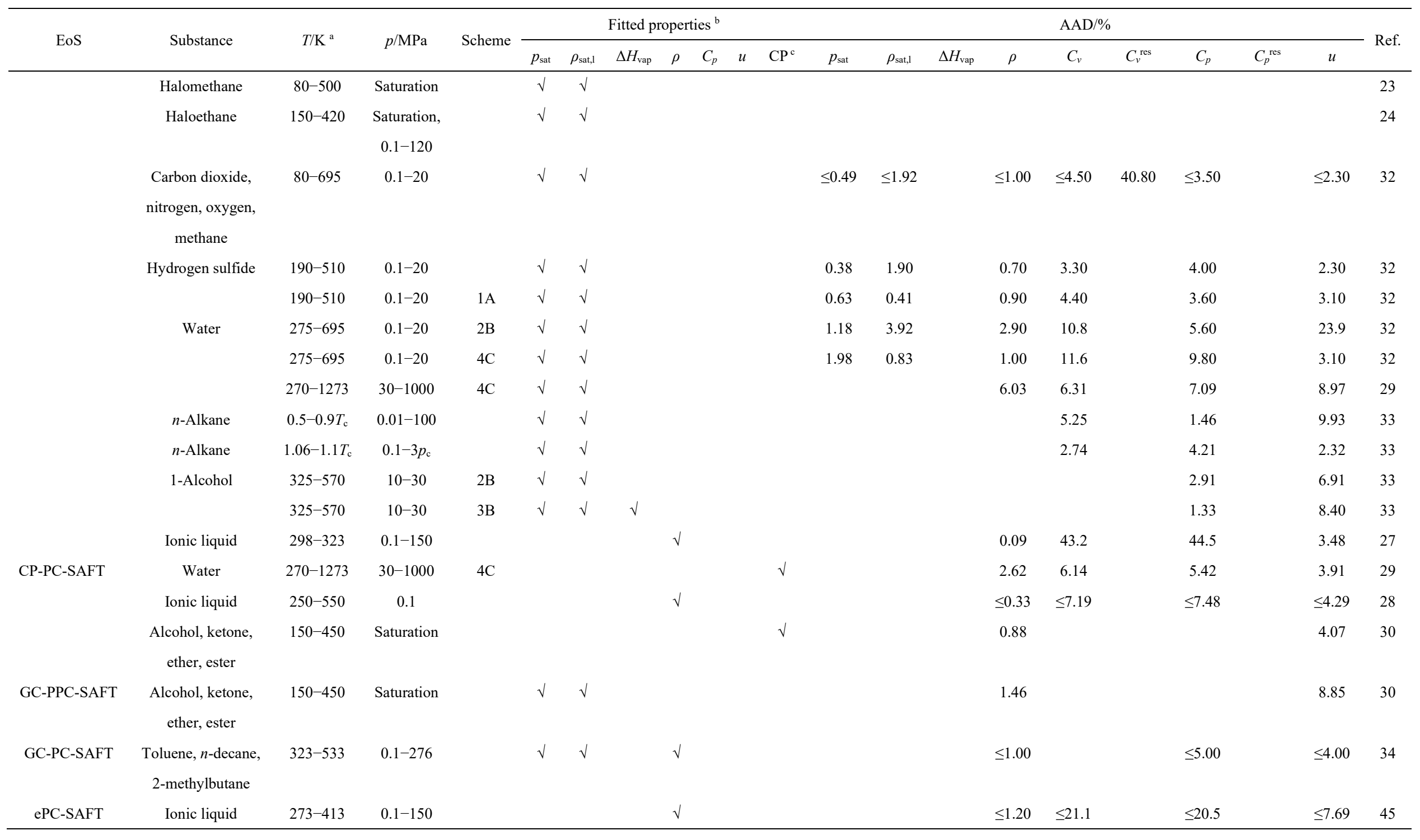




\begin{tabular}{|c|c|c|c|c|c|c|c|c|c|c|c|c|c|c|c|c|c|c|c|c|c|}
\hline \multirow{2}{*}{ EoS } & \multirow{2}{*}{ Substance } & \multirow{2}{*}{$T / \mathrm{K}^{\mathrm{a}}$} & \multirow{2}{*}{$p / \mathrm{MPa}$} & \multirow{2}{*}{ Scheme } & \multicolumn{7}{|c|}{ Fitted properties ${ }^{b}$} & \multicolumn{9}{|c|}{$\mathrm{AAD} / \%$} & \multirow{2}{*}{ Ref. } \\
\hline & & & & & $p_{\text {sat }}$ & $\rho_{\text {sat, }, 1}$ & $\Delta H_{\text {vap }}$ & $\rho$ & $C_{p}$ & $u$ & $\mathrm{CP}^{\mathrm{c}}$ & $p_{\text {sat }}$ & $\rho_{\text {sat, } 1}$ & $\Delta H_{\text {vap }}$ & $\rho$ & $C_{v}$ & $C_{v}^{\text {res }}$ & $C_{p}$ & $C_{p}^{\text {res }}$ & $u$ & \\
\hline Modified & 1-Alcohol & $248-470$ & $0.1-12$ & $2 \mathrm{~B}$ & $\sqrt{ }$ & $\sqrt{ }$ & & & & & & $\leq 1.56$ & $\leq 0.29$ & $\leq 2.51$ & $\leq 0.22$ & & & $\leq 8.82$ & & $\leq 4.35$ & 37 \\
\hline \multirow[t]{2}{*}{ SAFT-VR-Mie } & $n$-Alkane & $100-413$ & $0.1-150$ & & v & $\sqrt{ }$ & & & & & & $\leq 1.86$ & $\leq 1.46$ & $\leq 4.09$ & $\leq 1.65$ & & & $\leq 4.73$ & & $\leq 2.60$ & 37 \\
\hline & Perfluoroalkane & $150-400$ & $0.1-50$ & & $\sqrt{ }$ & $\sqrt{ }$ & & & & & & $\leq 0.92$ & $\leq 1.75$ & $\leq 1.48$ & $\leq 1.34$ & & & $\leq 0.92$ & & $\leq 7.38$ & 37 \\
\hline \multirow[t]{5}{*}{ SAFT-VR-Mie } & 1-Alcohol & $298-570$ & $0.1-30$ & $3 \mathrm{~B}$ & v & $\sqrt{ }$ & $\sqrt{ }$ & v & & v & & $\leq 1.70$ & $\leq 1.38$ & $\leq 1.86$ & $\leq 1.00$ & & & $\leq 1.40$ & 6.20 & $\leq 2.98$ & 35 \\
\hline & Alcohol & $333-421$ & $10-60$ & $3 \mathrm{~B}$ & $\sqrt{ }$ & $\sqrt{ }$ & $\sqrt{ }$ & $\sqrt{ }$ & & $\sqrt{ }$ & & $\leq 1.55$ & & $\leq 2.75$ & $\leq 0.63$ & & & $\leq 1.50$ & & $\leq 2.92$ & 35 \\
\hline & $n$-Alkane & $298-403$ & $0.1-100$ & & v & $v$ & & $\checkmark$ & & v & & $\leq 17.7$ & $\leq 1.19$ & & $\leq 1.17$ & & & $\leq 7.25$ & $\leq 33.3$ & $\leq 2.75$ & 36 \\
\hline & $n$-Alkane + & $303-308$ & 0.1 & $3 \mathrm{~B}$ & $\sqrt{ }$ & $\sqrt{ }$ & $\sqrt{ }$ & $\sqrt{ }$ & & 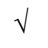 & & & & & $\leq 0.63$ & & & & & $\leq 4.96$ & 35 \\
\hline & 1-alcohol & & & & & & & & & & & & & & & & & & & & \\
\hline \multirow[t]{2}{*}{ SAFT-VR-SW } & $n$-Alkane & $298-403$ & $0.1-100$ & & $\sqrt{ }$ & $\sqrt{ }$ & & & & & & $\leq 58.2$ & $\leq 3.72$ & & $\leq 2.30$ & & & $\leq 8.72$ & $\leq 40.3$ & $\leq 16.5$ & 36 \\
\hline & FAME mixture & $300-700$ & 0.1 & & v & v & & $\sqrt{ }$ & & v & & & & & & & & & & & 38 \\
\hline \multirow[t]{6}{*}{ soft-SAFT } & $n$-Alkane & $0.7-0.9 T_{\mathrm{c}}$ & $0.1-30$ & & $\sqrt{ }$ & $\sqrt{ }$ & & & & & & $\leq 3.24$ & $\leq 1.73$ & & & & & $\leq 3.23$ & & & 39 \\
\hline & Perfluoroalkane & $0.7-0.9 T_{\mathrm{c}}$ & $0.1-30$ & & 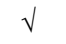 & $\sqrt{ }$ & & & & & & $\leq 2.71$ & $\leq 1.59$ & & & & & $\leq 6.21$ & & & 39 \\
\hline & 1-Achohol & $300-500$ & $0.1-30$ & $2 \mathrm{~B}$ & $\checkmark$ & $\sqrt{ }$ & & & $\sqrt{ }$ & & & $\leq 3.88$ & $\leq 1.63$ & & & & & $\leq 5.38$ & & & 39 \\
\hline & Water & $293-500$ & $0.1-50$ & $4 \mathrm{C}$ & $\sqrt{ }$ & $\sqrt{ }$ & & & $\sqrt{ }$ & & & 2.01 & 0.97 & & & & & 1.20 & & & 39 \\
\hline & $n$-Alkane & $1.1-1.5 T_{\mathrm{c}}$ & -- & & $\sqrt{ }$ & $\sqrt{ }$ & & & & & & & & & & $\leq 4.26$ & $\leq 55.9$ & $\leq 4.61$ & $\leq 24.0$ & & 40 \\
\hline & 1-Achohol & $280-573$ & $0.1-30$ & $2 \mathrm{~B}$ & $\sqrt{ }$ & $\sqrt{ }$ & & & & & & & & & & & & $\leq 18.1$ & $\leq 30.0$ & & 40 \\
\hline Crossover & $n$-Alkane & $1.1-1.5 T_{\mathrm{c}}$ & -- & & $\sqrt{ }$ & $\sqrt{ }$ & & & & & & & & & & & $\leq 38.2$ & & $\leq 13.5$ & & 40 \\
\hline \multirow[t]{5}{*}{ soft-SAFT } & Isobutene & $1.1-1.25 T_{\mathrm{c}}$ & -- & & $\sqrt{ }$ & $\sqrt{ }$ & & & & & & & & & & & & & & & 41 \\
\hline & Perfluoroethane & $225-500$ & $0.1-30$ & & $\sqrt{ }$ & $\sqrt{ }$ & & & & & & & & & & 5.00 & & 5.00 & & & 42 \\
\hline & $\begin{array}{c}\text { Propane + } \\
\text { isobutene }\end{array}$ & $280-330$ & 0.1 & & $\sqrt{ }$ & v & & & & & & & & & & 3.00 & & & & & 41 \\
\hline & $n$-Alkane + & $280-318$ & 0.1 & $2 \mathrm{~B}$ & $\sqrt{ }$ & $\sqrt{ }$ & & & & & & & & & & & & & & & 41 \\
\hline & 1-alcohol & & & & & & & & & & & & & & & & & & & & \\
\hline \multirow[t]{2}{*}{ SAFT-BACK } & $n$-Alkane & $150-675$ & -- & & $\checkmark$ & $\checkmark$ & & & & & $\sqrt{ }$ & & & & & 1.90 & & 1.20 & & 2.30 & 43 \\
\hline & $n$-Alkane mixture & $300-400$ & $0.1-300$ & & $\sqrt{ }$ & $\sqrt{ }$ & & & & & $\sqrt{ }$ & & & & $\leq 1.00$ & & & & & $\leq 3.50$ & 44 \\
\hline
\end{tabular}




\begin{tabular}{|c|c|c|c|c|c|c|c|c|c|c|c|c|c|c|c|c|c|c|c|c|c|}
\hline \multirow{2}{*}{ EoS } & \multirow{2}{*}{ Substance } & \multirow{2}{*}{$T / \mathrm{K}^{\mathrm{a}}$} & \multirow{2}{*}{$p / \mathrm{MPa}$} & \multirow{2}{*}{ Scheme } & \multicolumn{7}{|c|}{ Fitted properties ${ }^{\mathrm{b}}$} & \multicolumn{9}{|c|}{$\mathrm{AAD} / \%$} & \multirow{2}{*}{ Ref. } \\
\hline & & & & & $p_{\text {sat }}$ & $\rho_{\text {sat }, 1}$ & $\Delta H_{\text {vap }}$ & $\rho$ & $C_{p}$ & $u$ & $\mathrm{CP}^{\mathrm{c}}$ & $p_{\text {sat }}$ & $\rho_{\text {sat }, 1}$ & $\Delta H_{\text {vap }}$ & $\rho$ & $C_{v}$ & $C_{v}^{\text {res }}$ & $C_{p}$ & $C_{p}^{\text {res }}$ & $u$ & \\
\hline \multirow[t]{10}{*}{ SAFT + Cubic } & Halomethane & $80-500$ & Saturation & & $\sqrt{ }$ & $\sqrt{ }$ & & & & & $\sqrt{ }$ & & & & & & & & & & 23 \\
\hline & Haloethane & $150-420$ & $\begin{array}{c}\text { Saturation, } \\
0.1-120\end{array}$ & & $\sqrt{ }$ & $\sqrt{ }$ & & & & & $\sqrt{ }$ & & & & & & & & & & 24 \\
\hline & Water, methanol & $150-1400$ & $\begin{array}{c}\text { Saturation, } \\
0.1-1000\end{array}$ & $4 \mathrm{C}, 3 \mathrm{~B}$ & $\sqrt{ }$ & $\sqrt{ }$ & & & & & $\sqrt{ }$ & & & & & & & & & & 15 \\
\hline & $\begin{array}{l}\text { Carbon dioxide, } \\
n \text {-pentadecane }\end{array}$ & $200-1200$ & $\begin{array}{c}\text { Saturation, } \\
0.1-800\end{array}$ & & $\sqrt{ }$ & $\sqrt{ }$ & & & & & $\sqrt{ }$ & & & & & & & & & & 15 \\
\hline & $n$-Hexane & $243-503$ & $\begin{array}{c}\text { Saturation, } \\
0.1-1000\end{array}$ & & & $\sqrt{ }$ & & & & & $\sqrt{ }$ & & & & & & & & & & 26 \\
\hline & $\begin{array}{l}\text { Di-isodecyl } \\
\text { phthalate }\end{array}$ & $293-413$ & $0.1-160$ & & & & & & & & $\sqrt{ }$ & & & & & & & & & & 21 \\
\hline & 1-Alkene & $298-433$ & $0.1-240$ & & $\sqrt{ }$ & $\sqrt{ }$ & & & & & $\sqrt{ }$ & & & & & & & & & & 22 \\
\hline & $n$-Octane & $299-385$ & $0.1-1000$ & & & $\sqrt{ }$ & & & & & $\sqrt{ }$ & & & & & & & & & & 25 \\
\hline & $\begin{array}{c}n \text {-Octane }+ \\
n \text {-hexadecane }\end{array}$ & $313-433$ & $0.1-120$ & & & $\sqrt{ }$ & & & & & $\sqrt{ }$ & & & & & & & & & & 25 \\
\hline & Ionic liquid & $298-323$ & $0.1-150$ & & & & & $\sqrt{ }$ & & & & & & & 0.10 & 2.06 & & 3.10 & & 1.34 & 27 \\
\hline VTPR & $\begin{array}{c}\text { Alkane, ketone, } \\
\text { water, etc. }\end{array}$ & $90-480$ & Saturation & & $\sqrt{ }$ & & & & $\sqrt{ }$ & & & 0.90 & & 1.21 & & & & 0.78 & & & 46 \\
\hline SBWR & $n$-Hexane & $243-503$ & $\begin{array}{c}\text { Saturation, } \\
0.1-1000\end{array}$ & & $\sqrt{ }$ & $\sqrt{ }$ & & & & & & & & & & & & & & & 26 \\
\hline PHTC & FAEE & $286-341$ & $0.1-100$ & & & & & $\sqrt{ }$ & & & & & & & 0.11 & & & 0.91 & & 2.71 & 47 \\
\hline
\end{tabular}

$413{ }^{a}$ Temperature and pressure ranges are only for heat capacities; ${ }^{b}$ Properties used in parameter estimation; ${ }^{\mathrm{c}} \mathrm{CP}$ : critical point. 


\section{Comparisons of Literature Models for Selected Compounds}

415 Following our discussion of the general performance of each EoS, it is of relevance to 416 comparatively evaluate the different models for specific compounds. Table 3 classifies the 417 EoS according to specific families of compounds of $n$-alkanes, 1 -alcohols, water, and other 418 compounds (carbon dioxide and hydrogen sulfide). In Table 3, different temperature and 419 pressure ranges studied with different EoS are shown, and this makes it hard to give an 420 overall comparison of all models. Therefore, in this work, only the EoS used at the similar 421 conditions will be discussed.

\section{$422 \quad 3.1$ n-Alkanes}

423 For $n$-alkanes at evaluated pressures, the accuracy of CPA, original SAFT and PC-SAFT 424 were compared. ${ }^{33}$ The results show that the $C_{v}$ data are best described by the original SAFT, 425 and the most accurate $C_{p}$ and $u$ results can be obtained with CPA and PC-SAFT, 426 respectively. Overall, the original SAFT EoS has the best performance for heat capacities 427 with the AAD of both $C_{v}$ and $C_{p}$ lower than 5\%, while its accuracy of $u$ is the lowest among 428 the three EoS. In the near critical region $\left(T_{\mathrm{r}}=1.06-1.10\right)$, all three EoS have satisfactory 429 accuracy for heat capacities, while the AAD of $u$ calculated by the original SAFT is 430 relatively large, reaching to about $6.6 \%$. From Table 3 , we can conclude that PC-SAFT 431 gives a best balance between the heat capacities and sound velocity, but none of the three 432 EoS can simultaneously give satisfactory descriptions of all of these properties. Therefore, 433 some attempts, such as re-estimating the parameters and even universal constants for PC434 SAFT with the $C_{p}$ or $u$ data may be of interest. ${ }^{60}$ 
435 Two SAFT-VR models with the SW and Mie potentials were used to calculate the 436 properties of $n$-alkanes. ${ }^{36}$ The SAFT-VR-Mie EoS is superior to SAFT-VR-SW for all 437 studied properties, especially $p_{\text {sat }}$ and $u$. The high accuracy of $u$ with the former model may 438 be due to including $u$ data in the parameter estimation procedure, but this method cannot 439 improve the predictive ability for $C_{p}$ remarkably. However, when a higher-order 440 perturbation term is included in SAFT-VR-Mie, the performance for both VLE and 441 derivative properties can be improved significantly. ${ }^{37}$ Specially, although the parameters 442 of modified SAFT-VR-Mie are only fitted to $p_{\text {sat }}$ and $\rho_{\text {sat }, 1}$, the AAD of $u$ can still be lower 443 than $2.6 \%$.

444 The soft-SAFT EoS can give rather good performance for $C_{v}$ and $C_{p}$, while the deviations 445 of their residual values are quite large. ${ }^{40}$ Coupling with the RG approach, a crossover soft446 SAFT EoS was also used to calculate the heat capacities of $n$-alkanes. The accuracy of $C_{v}{ }^{\text {res }}$ 447 and $C_{p}{ }^{\text {res }}$ at $T_{\mathrm{r}}=1.1-1.5$ with this crossover EoS is obviously improved, while the AAD of $448 C_{v}{ }^{\text {res }}$ in the vicinity of the critical point $\left(T_{\mathrm{r}}=1.1\right)$ can still reach $11 \%$ for propane and $38 \%$ 449 for heptane, respectively. Such results indicate that a crossover equation may increase the 450 accuracy of residual heat capacities, but the basic performance of soft-SAFT may still pose 451 some limitations.

452 Finally, it can be seen that the SAFT-BACK EoS can describe well the heat capacities 453 and sound velocity of $n$-alkanes in both subcritical and supercritical regions, giving a best 454 performance for this kind of compounds among these EoS. 
456 For the properties of 1-alcohols, the original CPA and the modified CPA with 5457 parameter $\alpha$ function were tested..$^{20} \mathrm{~A}$ two-site association scheme (2B) was chosen, and 458 the parameters of two models were obtained from VLE properties, heat capacity, and 459 critical properties. In Table 3, we can see that the modified CPA shows similar performance 460 for VLE properties compared to the original CPA and much better performance for $C_{p}$ and $461 C_{p}{ }^{\text {res }}$. This indicates that a more flexible $\alpha$ function can increase the correlation ability of this type of EoS for heat capacities. In addition, the original CPA shows lower AAD of the saturated liquid density $\left(\rho_{\text {sat,l }}\right)$ for short-chain alcohols than the modified model, while the deviations increase with the increase of carbon numbers. up to $30 \mathrm{MPa}$ with two different association schemes is compared. ${ }^{33}$ The best balance between $C_{p}$ and $u$ is given by PC-SAFT, which gives an AAD of $u$ lower than $9 \%$ with both the $2 \mathrm{~B}$ and $3 \mathrm{~B}$ schemes. Generally, the three models using the $3 \mathrm{~B}$ scheme have lower AAD of $C_{p}$ but higher AAD of $u$ compared to $2 \mathrm{~B}$. The only exception is with original SAFT for $C_{p}$, with which the $2 \mathrm{~B}$ model provides higher accuracy. In addition, it should be noticed that the original CPA with the $3 \mathrm{~B}$ scheme provides a rather satisfactory performance on $C_{p}$, with the AAD of $0.9 \%$. This may be because the parameters of the EoS are correlated not only to the VLE properties, but also to the $C_{p}$ data. However, as mentioned by de Villiers

474 et al., ${ }^{33}$ the same parameter regression strategy applied to the original SAFT and PC-SAFT 475 cannot give the same improvements compared to CPA. In conclusion, it should be realized 476 that although a more rigorous association scheme (3B) for 1-alcohols is used, the higher 
477 accuracy of the calculated $C_{p}$ and $u$ can still not be obtained simultaneously, indicating the

478 limitations of the EoS. Furthermore, it can be seen that one parameter regression strategy

479 for different EoS might have different effects on properties of fluids, thus the selection of

480 the most suitable fitting procedure shall depend on the equation of state used, when going

481 beyond vapor pressure and liquid density

482 By including VLE, compressed $\rho$ and $u$ data in the parameter estimation, SAFT-VR-Mie

483 provides an excellent representation of these properties. ${ }^{35}$ Unlike $n$-alkanes, both the

484 calculated $C_{p}$ and $C_{p}{ }^{\text {res }}$ for 1-alcohols have rather low deviations from the experimental

485 data, and the overall performance for $u$ and $C_{p}$ is better than PC-SAFT. With model

486 parameters only correlated to VLE properties, the modified SAFT-VR-Mie with a higher-

487 order perturbation term can still give acceptable performance for $C_{p}$ and $u$, with methanol

488 for $C_{p}$ being the only exception. ${ }^{37}$ But it should be pointed out that the calculated $C_{p}$ for

489 methanol with original SAFT-VR-Mie in ref. 16 is only at one point, while the results with

490 the modified EoS are obtained over wider temperature and pressure ranges. The

491 performance of soft-SAFT with two different parametrization strategies ${ }^{40}$ is also compared

492 in Table 3. The soft-SAFT EoS seems to have worse accuracy of $C_{p}$ than the most

493 aforementioned EoS, no matter whether the $C_{p}$ data are used in the parametrization process.

4943.3 Water

495 Compared with the original CPA EoS, the modified CPA proposed by Palma et al. gives 496 much higher accuracy for the $C_{p}$ for water, while the AAD of $\rho_{\text {sat, }, 1}$ increases as the data of 497 this property are not included in the fitting procedure. ${ }^{19}$ In Table 3 are also presented and 
compared the saturated properties of water obtained from the original CPA and PC-SAFT

499 at reduced temperatures up to $0.96 .{ }^{31}$ Both EoS can accurately reproduce the VLE data.

500 The original CPA is superior to PC-SAFT for all the investigated properties, especially for

501 the residual heat capacities and speed of sound. Nevertheless, both CPA and PC-SAFT

502 cannot give satisfactory results of these second-order derivative properties, and the $C_{v}$ and

$503 u$ data are even not qualitatively described. Finally, the VTPR EoS gives similar AAD of

$504 C_{p}$ compared to the modified CPA. ${ }^{46}$

505 For water at evaluated pressures up to $20 \mathrm{MPa}$, two different association schemes (2B

506 and 4C) with the original SAFT and PC-SAFT were tested by estimating its thermophysical

507 properties. $^{32}$ The original SAFT with the 4C scheme has better performance for all the

508 studied properties compared to the 2B scheme, while the descriptions of heat capacities

509 and sound velocity are still unsatisfactory. The PC-SAFT EoS with the 4C scheme shows

510 higher accuracy for $C_{v}$ and $u$ than with the original SAFT. Furthermore, PC-SAFT provides

511 higher AAD of heat capacities with 4C scheme than with $2 \mathrm{~B}$ scheme. Therefore, for water,

512 a similar conclusion as for 1-alcohols' can be made: A more rigorous association scheme

513 is not a sufficient condition for better descriptions of thermophysical properties, as the

514 accuracy of the calculated results is also deeply influenced by the selected EoS itself.

515 Considering the 4C scheme, the performance of PC-SAFT and CP-PC-SAFT for water was

516 compared over a wide temperature and pressure ranges $(T=270-1273 \mathrm{~K}, p=30-1000$

$517 \mathrm{MPa}) .{ }^{29}$ It can be concluded that the latter is superior to the former for all properties at the

518 compressed regions, while CP-PC-SAFT is a worse estimator than PC-SAFT for saturated 
density near the critical point. Finally, the soft-SAFT EoS with parameters fitted to VLE

520 and $C_{p}$ reproduces rather well these properties, with the AAD lower than $2.1 \% .{ }^{39}$ However,

521 a wider temperature range still needs to be considered for soft-SAFT in order to compare

522 its performance for $C_{p}$ with the original SAFT and PC-SAFT.

\section{3.4 Other Compounds}

524 Except for $n$-alkanes, 1-alcohols and water, the calculated heat capacities of some other

525 fluids are also compared using various equations of state. The properties of carbon dioxide,

526 nitrogen, oxygen and methane were estimated with the original SAFT and PC-SAFT

527 models, ${ }^{32}$ and the results demonstrate that the latter model has better performance for most

528 considered properties. The only exception is for saturated liquid density, with the AAD of

$529 \quad 1.9 \%$ for PC-SAFT and $1.8 \%$ for SAFT, respectively.

530 Using the original SAFT and PC-SAFT, the properties of hydrogen sulfide were

531 calculated with two different association schemes. ${ }^{32}$ It can be seen that PC-SAFT is

532 superior to the original SAFT for all properties no matter whether the association is

533 considered or not. As for the effect of the association scheme, compared with the non-

534 associating model, the original SAFT EoS with the 1A scheme shows a small effect on

535 VLE properties and compressed $\rho$. The AAD using the 1A scheme provides better accuracy

536 of $C_{v}$ and worse accuracy of $C_{p}$ and $u$ than without considering the association contribution.

537 Unlike the original SAFT EoS, PC-SAFT combined with the 1A scheme of hydrogen

538 sulfide has similar performance for most properties compared to using the non-associating

539 model, even though more parameters are involved in the former one. 
540 For the performance for ILs, five EoS have been tested in the literature. ${ }^{27,28,45}$ Different

541 contributions to Helmholtz energy are considered in each model: For CPA, ILs are regarded

542 as associating compounds with $2 \mathrm{~B}$ scheme. Five positive sites and five negative sites are

543 considered in the association contribution of PC-SAFT. No association term is included in

544 the SAFT + Cubic, CP-PC-SAFT, and ePC-SAFT EoS for ILs, while the Coulomb

545 interaction contribution is considered in the last model. Overall, at the pressures up to 150

546 MPa, SAFT + Cubic shows the best performance for both heat capacities and speed of

547 sound, and PC-SAFT and CPA give the lowest accuracy of heat capacity and speed of sound,

548 respectively. The description of heat capacities with ePC-SAFT is also not satisfactory,

549 with the AAD lower than $21 \%$. It is worth mentioning that, with comparison to CP-PC-

550 SAFT, the performance of ePC-SAFT for ILs at the atmospheric pressure is still worse. ${ }^{28}$

551 Some conclusions are drawn from the above discussion. First, although the PC-SAFT

552 EoS may not give the best results in some cases, it exhibits a better overall performance for

553 investigated properties (heat capacities, speed of sound, VLE, and compressed density) and

554 fluids than the original CPA and SAFT. Second, a more rigorous association scheme may

555 not always lead to a better description of thermophysical properties, as the accuracy

556 depends a lot on the overall model (EoS) considered. Finally, the parametrization method

557 where $C_{p}$ or $u$ data are used in the parameter estimation cannot always improve the accuracy

558 of calculated second-order derivative properties. On the other hand, we can state based on

559 the literature studies that for the models of VTPR, CPA, and SAFT-VR-Mie, very good

560 results are obtained by including the $C_{p}$ or $u$ data in the parameter estimation procedure. 


\begin{tabular}{|c|c|c|c|c|c|c|c|c|c|c|c|c|c|c|c|c|c|c|c|c|c|}
\hline \multirow{2}{*}{ Substance } & \multirow{2}{*}{ EoS } & \multirow{2}{*}{$T / \mathrm{K}^{\mathrm{a}}$} & \multirow{2}{*}{$p / \mathrm{MPa}$} & \multirow{2}{*}{ scheme } & \multicolumn{7}{|c|}{ Fitted properties ${ }^{\mathrm{b}}$} & \multicolumn{9}{|c|}{$\mathrm{AAD} / \%$} & \multirow{2}{*}{ Ref. } \\
\hline & & & & & $p_{\text {sat }}$ & $\rho_{\text {sat }, 1}$ & $\Delta H_{\text {vap }}$ & $\rho$ & $C_{p}$ & $u$ & $\mathrm{CP}^{\mathrm{c}}$ & $p_{\text {sat }}$ & $\rho_{\text {sat } 1}$ & $\Delta H_{\text {vap }}$ & $\rho$ & $C_{v}$ & $C_{v}^{\text {res }}$ & $C_{p}$ & $C_{p}^{\text {res }}$ & $u$ & \\
\hline \multirow[t]{15}{*}{$n$-Alkane } & $\mathrm{CPA}$ & $0.5-0.9 T_{\mathrm{c}}$ & $0.01-100$ & & $\sqrt{ }$ & $\sqrt{ }$ & & & & & & & & & & 5.50 & & 3.24 & & 9.31 & 33 \\
\hline & & $1.06-1.1 T_{\mathrm{c}}$ & $0.1-3 p_{\mathrm{c}}$ & & $\sqrt{ }$ & $\sqrt{ }$ & & & & & & & & & & 2.02 & & 3.46 & & 3.43 & 33 \\
\hline & SAFT & $0.5-0.9 T_{\mathrm{c}}$ & $0.01-100$ & & $\sqrt{ }$ & $\sqrt{ }$ & & & & & & & & & & 3.04 & & 4.33 & & 17.6 & 33 \\
\hline & & $1.06-1.1 T_{\mathrm{c}}$ & $0.1-3 p_{\mathrm{c}}$ & & $\sqrt{ }$ & $\sqrt{ }$ & & & & & & & & & & 2.79 & & 3.61 & & 6.58 & 33 \\
\hline & PC-SAFT & $0.5-0.9 T_{\mathrm{c}}$ & $0.01-100$ & & $\sqrt{ }$ & $\sqrt{ }$ & & & & & & & & & & 5.25 & & 1.46 & & 9.93 & 33 \\
\hline & & $1.06-1.1 T_{\mathrm{c}}$ & $0.1-3 p_{\mathrm{c}}$ & & $\sqrt{ }$ & $\sqrt{ }$ & & & & & & & & & & 2.74 & & 4.21 & & 2.32 & 33 \\
\hline & Modified & $100-413$ & $0.1-150$ & & $\sqrt{ }$ & $\sqrt{ }$ & & & & & & $\leq 1.86$ & $\leq 1.46$ & $\leq 4.09$ & $\leq 1.65$ & & & $\leq 4.73$ & & $\leq 2.60$ & 37 \\
\hline & SAFT-VR-Mie & & & & & & & & & & & & & & & & & & & & \\
\hline & SAFT-VR-Mie & $298-403$ & $0.1-100$ & & $\sqrt{ }$ & $\sqrt{ }$ & & $\sqrt{ }$ & & $\sqrt{ }$ & & $\leq 17.7$ & $\leq 1.19$ & & $\leq 1.17$ & & & $\leq 7.25$ & $\leq 33.3$ & $\leq 2.75$ & 36 \\
\hline & SAFT-VR-SW & $298-403$ & $0.1-100$ & & $\sqrt{ }$ & $\sqrt{ }$ & & & & & & $\leq 58.2$ & $\leq 3.72$ & & $\leq 2.30$ & & & $\leq 8.72$ & $\leq 40.3$ & $\leq 16.5$ & 36 \\
\hline & soft-SAFT & $0.7-0.9 T_{\mathrm{c}}$ & $0.1-30$ & & $\sqrt{ }$ & $\sqrt{ }$ & & & & & & $\leq 3.24$ & $\leq 1.73$ & & & & & $\leq 3.23$ & & & 39 \\
\hline & & $1.1-1.5 T_{\mathrm{c}}$ & -- & & $\sqrt{ }$ & $\sqrt{ }$ & & & & & & & & & & $\leq 4.26$ & $\leq 55.9$ & $\leq 4.61$ & $\leq 24.0$ & & 40 \\
\hline & Crossover & $1.1-1.5 T_{\mathrm{c}}$ & -- & & $\sqrt{ }$ & $\sqrt{ }$ & & & & & & & & & & & $\leq 38.2$ & & $\leq 13.5$ & & 40 \\
\hline & soft-SAFT & & & & & & & & & & & & & & & & & & & & \\
\hline & SAFT-BACK & $150-675$ & -- & & $\sqrt{ }$ & $\sqrt{ }$ & & & & & $\sqrt{ }$ & & & & & 1.90 & & 1.20 & & 2.30 & 43 \\
\hline \multirow[t]{8}{*}{ 1-Alcohol } & Modified CPA & $260-490$ & Saturation & $2 \mathrm{~B}$ & $\sqrt{ }$ & $\sqrt{ }$ & & & $\sqrt{ }$ & & $\sqrt{ }$ & $\leq 0.79$ & $\leq 1.94$ & $\leq 1.91$ & & & & $\leq 2.25$ & $\leq 5.52$ & & 20 \\
\hline & $\mathrm{CPA}$ & $260-490$ & Saturation & $2 \mathrm{~B}$ & $\sqrt{ }$ & $\sqrt{ }$ & & & $\sqrt{ }$ & & $\sqrt{ }$ & $\leq 0.95$ & $\leq 1.25$ & $\leq 1.75$ & & & & $\leq 5.31$ & $\leq 12.6$ & & 20 \\
\hline & & $325-570$ & $10-30$ & $2 \mathrm{~B}$ & $\sqrt{ }$ & $\sqrt{ }$ & & & & & & & & & & & & 4.07 & & 12.5 & 33 \\
\hline & & $325-570$ & $10-30$ & $3 \mathrm{~B}$ & $\sqrt{ }$ & $\sqrt{ }$ & $\sqrt{ }$ & & $\sqrt{ }$ & & & & & & & & & 0.93 & & 14.2 & 33 \\
\hline & SAFT & $325-570$ & $10-30$ & $2 \mathrm{~B}$ & $\sqrt{ }$ & $\sqrt{ }$ & & & & & & & & & & & & 2.17 & & 15.0 & 33 \\
\hline & & $325-570$ & $10-30$ & $3 \mathrm{~B}$ & $\sqrt{ }$ & $\sqrt{ }$ & $\sqrt{ }$ & & & & & & & & & & & 5.59 & & 16.0 & 33 \\
\hline & PC-SAFT & $325-570$ & $10-30$ & $2 \mathrm{~B}$ & $\sqrt{ }$ & $\sqrt{ }$ & & & & & & & & & & & & 2.91 & & 6.91 & 33 \\
\hline & & $325-570$ & $10-30$ & $3 \mathrm{~B}$ & $\sqrt{ }$ & $\sqrt{ }$ & $\sqrt{ }$ & & & & & & & & & & & 1.33 & & 8.40 & 33 \\
\hline
\end{tabular}




\begin{tabular}{|c|c|c|c|c|c|c|c|c|c|c|c|c|c|c|c|c|c|c|c|c|c|}
\hline \multirow{2}{*}{ Substance } & \multirow{2}{*}{ EoS } & \multirow{2}{*}{$T / \mathrm{K}^{\mathrm{a}}$} & \multirow{2}{*}{$p / \mathrm{MPa}$} & \multirow{2}{*}{ scheme } & \multicolumn{7}{|c|}{ Fitted properties ${ }^{b}$} & \multicolumn{9}{|c|}{$\mathrm{AAD} / \%$} & \multirow{2}{*}{ Ref } \\
\hline & & & & & $p_{\text {sat }}$ & $\rho_{\mathrm{sat}, 1}$ & $\Delta H_{\text {vap }}$ & $\rho$ & $C_{p}$ & $u$ & $\mathrm{CP}^{\mathrm{c}}$ & $p_{\text {sat }}$ & $\rho_{\text {sat }, 1}$ & $\Delta H_{\text {vap }}$ & $\rho$ & $C_{v}$ & $C_{v}^{\text {res }}$ & $C_{p}$ & $C_{p}^{\text {res }}$ & $u$ & \\
\hline \multirow{17}{*}{ Water } & Modified & $248-470$ & $0.1-12$ & $2 \mathrm{~B}$ & $\sqrt{ }$ & $\sqrt{ }$ & & & & & & $\leq 1.56$ & $\leq 0.29$ & $\leq 2.51$ & $\leq 0.22$ & & & $\leq 8.82$ & & $\leq 4.35$ & 37 \\
\hline & SAFT-VR-Mie & & & & & & & & & & & & & & & & & & & & \\
\hline & SAFT-VR-Mie & $298-570$ & $0.1-30$ & $3 \mathrm{~B}$ & $\sqrt{ }$ & $\sqrt{ }$ & $\sqrt{ }$ & $\sqrt{ }$ & & $\sqrt{ }$ & & $\leq 1.70$ & $\leq 1.38$ & $\leq 1.86$ & $\leq 1.00$ & & & $\leq 1.40$ & 6.20 & $\leq 2.98$ & 35 \\
\hline & soft-SAFT & $300-500$ & $0.1-30$ & 2B & $\sqrt{ }$ & $\sqrt{ }$ & & & $\sqrt{ }$ & & & $\leq 3.88$ & $\leq 1.63$ & & & & & $\leq 5.38$ & & & 39 \\
\hline & & $280-573$ & $0.1-30$ & $2 \mathrm{~B}$ & $\sqrt{ }$ & $\sqrt{ }$ & & & & & & & & & & & & $\leq 18.1$ & $\leq 30.0$ & & 40 \\
\hline & Modified CPA & $260-450$ & Saturation & $4 \mathrm{C}$ & $\sqrt{ }$ & & & & $\sqrt{ }$ & & $\sqrt{ }$ & 0.05 & 2.28 & 0.42 & & & & 0.84 & & & 19 \\
\hline & $\mathrm{CPA}$ & $260-450$ & Saturation & $4 \mathrm{C}$ & $\sqrt{ }$ & $\sqrt{ }$ & & & & & & 1.17 & 1.10 & 1.45 & & & & 7.43 & & & 19 \\
\hline & & $280-620$ & Saturation & $4 \mathrm{C}$ & $\sqrt{ }$ & $\sqrt{ }$ & & & & & & 0.75 & 1.16 & & & & 15.1 & & 11.0 & 9.05 & 31 \\
\hline & VTPR & $273-373$ & Saturation & & $\sqrt{ }$ & & & & $\sqrt{ }$ & & & 0.55 & & 0.85 & & & & 0.93 & & & 46 \\
\hline & SAFT & $275-695$ & $0.1-20$ & $2 \mathrm{~B}$ & $\sqrt{ }$ & $\sqrt{ }$ & & & & & & 1.19 & 3.08 & & 2.40 & 14.8 & & 7.80 & & 18.8 & 32 \\
\hline & & $275-695$ & $0.1-20$ & $4 \mathrm{C}$ & $\sqrt{ }$ & $\sqrt{ }$ & & & & & & 1.09 & 2.38 & & 1.80 & 13.4 & & 7.30 & & 8.50 & 32 \\
\hline & PC-SAFT & $280-620$ & Saturation & $4 \mathrm{C}$ & $\sqrt{ }$ & $\sqrt{ }$ & & & & & & 1.46 & 2.14 & & & & 21.8 & & 20.6 & 21.1 & 31 \\
\hline & & $275-695$ & $0.1-20$ & $2 \mathrm{~B}$ & $\sqrt{ }$ & $\sqrt{ }$ & & & & & & 1.18 & 3.92 & & 2.90 & 10.8 & & 5.60 & & 23.9 & 32 \\
\hline & & $275-695$ & $0.1-20$ & $4 \mathrm{C}$ & $\sqrt{ }$ & $\sqrt{ }$ & & & & & & 1.98 & 0.83 & & 1.00 & 11.6 & & 9.80 & & 3.10 & 32 \\
\hline & & $270-1273$ & $30-1000$ & $4 \mathrm{C}$ & $\sqrt{ }$ & $\sqrt{ }$ & & & & & & & & & 6.03 & 6.31 & & 7.09 & & 8.97 & 29 \\
\hline & CP-PC-SAFT & $270-1273$ & $30-1000$ & $4 \mathrm{C}$ & & & & & & & $\sqrt{ }$ & & & & 2.62 & 6.14 & & 5.42 & & 3.91 & 29 \\
\hline & soft-SAFT & $293-500$ & $0.1-50$ & $4 \mathrm{C}$ & $\sqrt{ }$ & $\sqrt{ }$ & & & $\sqrt{ }$ & & & 2.01 & 0.97 & & & & & 1.20 & & & 39 \\
\hline \multirow{2}{*}{$\begin{array}{l}\text { Carbon dioxide, } \\
\text { nitrogen, oxygen, } \\
\text { methane }\end{array}$} & SAFT & $80-695$ & $0.1-20$ & & $\sqrt{ }$ & $\sqrt{ }$ & & & & & & $\leq 1.58$ & $\leq 1.84$ & & $\leq 2.20$ & $\leq 5.90$ & 48.8 & $\leq 8.50$ & & $\leq 5.10$ & 32 \\
\hline & PC-SAFT & $80-695$ & $0.1-20$ & & $\sqrt{ }$ & $\sqrt{ }$ & & & & & & $\leq 0.49$ & $\leq 1.92$ & & $\leq 1.00$ & $\leq 4.50$ & 40.8 & $\leq 3.50$ & & $\leq 2.30$ & 32 \\
\hline \multirow[t]{4}{*}{ Hydrogen sulfide } & SAFT & $190-510$ & $0.1-20$ & & $\sqrt{ }$ & $\sqrt{ }$ & & & & & & 1.80 & 2.11 & & 1.60 & 6.10 & & 6.90 & & 3.70 & 32 \\
\hline & & $190-510$ & $0.1-20$ & $1 \mathrm{~A}$ & $\sqrt{ }$ & $\sqrt{ }$ & & & & & & 2.19 & 2.76 & & 1.20 & 4.70 & & 9.10 & & 8.60 & 32 \\
\hline & PC-SAFT & $190-510$ & $0.1-20$ & & $\sqrt{ }$ & $\sqrt{ }$ & & & & & & 0.38 & 1.90 & & 0.70 & 3.30 & & 4.00 & & 2.30 & 32 \\
\hline & & $190-510$ & $0.1-20$ & $1 \mathrm{~A}$ & $\sqrt{ }$ & $\sqrt{ }$ & & & & & & 0.63 & 0.41 & & 0.90 & 4.40 & & 3.60 & & 3.10 & 32 \\
\hline
\end{tabular}




\begin{tabular}{|c|c|c|c|c|c|c|c|c|c|c|c|c|c|c|c|c|c|c|c|c|c|}
\hline \multirow{2}{*}{ Substance } & \multirow{2}{*}{ EoS } & \multirow{2}{*}{$T / \mathrm{K}^{\mathrm{a}}$} & \multirow{2}{*}{$p / \mathrm{MPa}$} & \multirow{2}{*}{ scheme } & \multicolumn{7}{|c|}{ Fitted properties ${ }^{b}$} & \multicolumn{9}{|c|}{$\mathrm{AAD} / \%$} & \multirow{2}{*}{ Ref. } \\
\hline & & & & & $p_{\text {sat }}$ & $\rho_{\text {sat }, 1}$ & $\Delta H_{\text {vap }}$ & $\rho$ & $C_{p}$ & $u$ & $\mathrm{CP}^{\mathrm{c}}$ & $p_{\text {sat }}$ & $\rho_{\text {sat }, 1}$ & $\Delta H_{\text {vap }}$ & $\rho$ & $C_{v}$ & $C_{v}^{\text {res }}$ & $C_{p}$ & $C_{p}^{\mathrm{res}}$ & $u$ & \\
\hline \multirow[t]{5}{*}{ Ionic liquid } & $\mathrm{CPA}$ & $298-323$ & $0.1-150$ & $2 \mathrm{~B}$ & $\sqrt{ }$ & & & $\sqrt{ }$ & & & & & & & 0.95 & 6.55 & & 15.8 & & 21.0 & 27 \\
\hline & PC-SAFT & $298-323$ & $0.1-150$ & & & & & $\sqrt{ }$ & & & & & & & 0.09 & 43.2 & & 44.5 & & 3.48 & 27 \\
\hline & SAFT + Cubic & $298-323$ & $0.1-150$ & & & & & $\sqrt{ }$ & & & & & & & 0.10 & 2.06 & & 3.10 & & 1.34 & 27 \\
\hline & ePC-SAFT & $273-413$ & $0.1-150$ & & & & & $\sqrt{ }$ & & & & & & & $\leq 1.20$ & $\leq 21.1$ & & $\leq 20.5$ & & $\leq 7.69$ & 45 \\
\hline & CP-PC-SAFT & $250-550$ & 0.1 & & & & & $\sqrt{ }$ & & & & & & & $\leq 0.33$ & $\leq 7.19$ & & $\leq 7.48$ & & $\leq 4.29$ & 28 \\
\hline
\end{tabular}

$562{ }^{a}$ Temperature and pressure ranges are only for heat capacities; ${ }^{b}$ Properties used in parameter estimation; ${ }^{\mathrm{c}} \mathrm{CP}$ : critical point. 
571 density data from the DIPPR correlations ${ }^{61}$ at reduced temperatures from 0.5 to 0.9 . The

572 ideal gas isobaric heat capacity data required in the calculations are also obtained from the

573 DIPPR correlations with the uncertainties mostly lower than 1\% (reported by DIPPR).

\section{$574 \quad 4.1$ Compressed Region}

575 Table 4 presents the AAD of calculated heat capacities, $\mathrm{d} p / \mathrm{d} V$, and $\mathrm{d} p / \mathrm{d} T$ compared to 576 the values from NIST ${ }^{58}$ for some non-associating fluids at pressures up to $100 \mathrm{MPa}$. From

577 Table 4, it can be seen that, in general, the PC-SAFT EoS has better performance for both $578 C_{v}$ and $C_{p}$ with the overall AAD of $1.2 \%$ and $3.2 \%$, respectively (compared to 2.3 and $4.3 \%$

579 for CPA). It can also be noted that, for both models, the AAD of $C_{p}$ are commonly lower

580 than those of $C_{v}$ over a wide pressure range, which is consistent with the literature results

581 from Tables 2 and 3. The other two derivative properties in eq 2 , i.e. $\mathrm{d} p / \mathrm{d} V$ and $\mathrm{d} p / \mathrm{d} T$ are

582 also calculated and presented in Table 4. We can see that the AAD of these two properties

583 with both CPA and PC-SAFT are rather high (10-15\%), with PC-SAFT performing better. 
584 Such better descriptions of $\mathrm{d} p / \mathrm{d} V$ with PC-SAFT may be attributed to the more appropriate

585 co-volume values established by SAFT models than by the cubic equations. ${ }^{62,63}$ The largest

586 deviation of the calculated $\mathrm{d} p / \mathrm{d} V$ and $\mathrm{d} p / \mathrm{d} T$ observed in Table 4 is the AAD of $\mathrm{d} p / \mathrm{d} V$ for

587 2-methylpentane with CPA, reaching to $44 \%$, while the AAD of $C_{p}$ for this substance with

588 CPA is only $3.6 \%$. This is actually not surprising since the average ratio of $C_{p}$ id $/ C_{p}$ is higher

589 than $80 \%$ at the investigated conditions, and this means that the accuracy of the calculated

590 heat capacity depends more on the ideal gas contribution, as we have discussed in the

591 previous section.

592 Table 4. AAD of heat capacities for non-associating compounds with CPA and PC-SAFT

\begin{tabular}{ccccccccccccc}
\hline \multirow{2}{*}{ Compound } & \multirow{2}{*}{$T / \mathrm{K}$} & \multirow{2}{*}{$p / \mathrm{MPa}$} & \multicolumn{4}{c}{$\mathrm{CPA} / \%$} & \multicolumn{4}{c}{ PC-SAFT/\% } & \multirow{2}{*}{$\begin{array}{c}\text { No. of } \\
\text { points }\end{array}$} \\
\cline { 5 - 11 } Methane & $100-400$ & $0.1-100$ & 0.86 & 3.25 & 6.71 & 4.64 & 1.44 & 2.76 & 3.16 & 3.07 & 134 \\
Ethane & $200-500$ & $0.1-70$ & 1.46 & 4.67 & 9.70 & 9.11 & 1.44 & 4.00 & 5.02 & 5.12 & 105 \\
Propane & $200-500$ & $0.1-100$ & 3.06 & 4.18 & 10.7 & 12.4 & 1.04 & 4.93 & 8.12 & 9.29 & 147 \\
Butane & $250-550$ & $0.1-65$ & 1.95 & 4.71 & 9.41 & 13.5 & 1.24 & 4.11 & 8.90 & 9.08 & 98 \\
Pentane & $300-600$ & $0.1-100$ & 1.40 & 4.63 & 11.7 & 16.7 & 0.58 & 3.25 & 10.2 & 11.8 & 147 \\
Hexane & $300-600$ & $0.1-100$ & 2.07 & 4.01 & 13.7 & 18.3 & 0.64 & 3.09 & 9.69 & 12.0 & 147 \\
Heptane & $300-600$ & $0.1-100$ & 2.26 & 3.94 & 15.6 & 20.7 & 0.52 & 2.71 & 12.7 & 14.0 & 147 \\
Octane & $300-600$ & $0.1-100$ & 2.29 & 3.61 & 18.4 & 20.3 & 0.55 & 2.04 & 12.3 & 13.7 & 147 \\
Nonane & $250-550$ & $0.1-100$ & 3.22 & 4.76 & 23.0 & 25.9 & 0.54 & 3.44 & 15.1 & 18.4 & 136 \\
Decane & $350-650$ & $0.1-100$ & 1.66 & 4.36 & 20.0 & 23.7 & 0.63 & 2.05 & 13.7 & 15.4 & 147 \\
Dodecane & $400-700$ & $0.1-100$ & 2.25 & 2.93 & 22.7 & 23.8 & 1.42 & 0.96 & 11.3 & 14.2 & 147 \\
Isobutane & $250-550$ & $0.1-35$ & 1.76 & 3.91 & 13.5 & 14.2 & 1.18 & 3.31 & 8.82 & 7.72 & 56 \\
2-Methylpentane & $250-550$ & $0.1-100$ & 3.56 & 4.20 & 44.2 & 7.76 & 0.68 & 4.14 & 13.2 & 15.7 & 147 \\
Cyclohexane & $400-700$ & $0.1-80$ & 2.05 & 2.71 & 11.1 & 16.9 & 1.51 & 1.77 & 11.3 & 10.7 & 119 \\
Propylene & $200-500$ & $0.1-100$ & 2.41 & 5.68 & 12.0 & 11.7 & 1.14 & 5.20 & 6.36 & 8.61 & 147 \\
Benzene & $300-600$ & $0.1-75$ & 3.23 & 6.12 & 11.6 & 19.7 & 1.95 & 5.18 & 9.14 & 12.7 & 103 \\
Toluene & $400-700$ & $0.1-100$ & 1.66 & 3.56 & 11.2 & 17.1 & 0.78 & 2.33 & 10.2 & 10.3 & 147 \\
Carbon dioxide & $250-550$ & $0.1-100$ & 3.46 & 5.46 & 9.51 & 7.78 & 4.42 & 3.63 & 4.16 & 4.62 & 147 \\
\hline
\end{tabular}




\begin{tabular}{|c|c|c|c|c|c|c|c|c|c|c|c|}
\hline \multirow{2}{*}{ Compound } & \multirow{2}{*}{$T / \mathrm{K}$} & \multirow{2}{*}{$p / \mathrm{MPa}$} & \multicolumn{4}{|c|}{$\mathrm{CPA} / \%$} & \multicolumn{4}{|c|}{ PC-SAFT/\% } & \multirow{2}{*}{$\begin{array}{l}\text { No. of } \\
\text { points }\end{array}$} \\
\hline & & & $C_{p}$ & $C_{v}$ & $\mathrm{~d} p / \mathrm{d} V$ & $\mathrm{~d} p / \mathrm{d} T$ & $C_{p}$ & $C_{v}$ & $\mathrm{~d} p / \mathrm{d} V$ & $\mathrm{~d} p / \mathrm{d} T$ & \\
\hline Nitrogen & $100-400$ & $0.1-100$ & 1.72 & 4.56 & 5.98 & 4.11 & 1.47 & 3.07 & 2.18 & 2.45 & 147 \\
\hline Overall & & & 2.25 & 4.26 & 15.1 & 15.3 & 1.20 & 3.22 & 9.32 & 10.7 & \\
\hline
\end{tabular}

593 Figure 2 compares the four calculated derivative properties for dodecane from the 594 models and the values from NIST. It can be seen that the PC-SAFT EoS tends to 595 overestimate $\mathrm{d} p / \mathrm{d} V$, while the CPA EoS usually underestimates this property at high 596 pressures. As for $\mathrm{d} p / \mathrm{d} T$, both EoS give lower values than the values from NIST with PC597 SAFT being more accurate than CPA. It can also be observed in Figure $2 \mathrm{c}$ that both CPA 598 and PC-SAFT show satisfactory performance for the calculation of $C_{v}$, again with PC599 SAFT being the better of the two. In Figure 2d, Similar results are also obtained for $C_{p}$, for 600 which the PC-SAFT EoS shows higher accuracy than CPA, especially at low temperatures.

601 For supercritical fluids, the tendency of $C_{p}$ can be well described by CPA and PC-SAFT, 602 although there are some deviations at the pseudocritical temperatures. However, both 603 models fail to capture the $C_{v}$ maxima at the supercritical conditions, and these results can 604 also be obtained for other compounds, such as toluene, as shown in Figure 3. In fact, the 605 failure in the description of $C_{v}$ maxima is quite common for analytical models. ${ }^{64}$ From 606 Figures 2 and 3, we can conclude again that the PC-SAFT EoS has better performance than 607 CPA for all four derivative properties for non-associating fluids. 


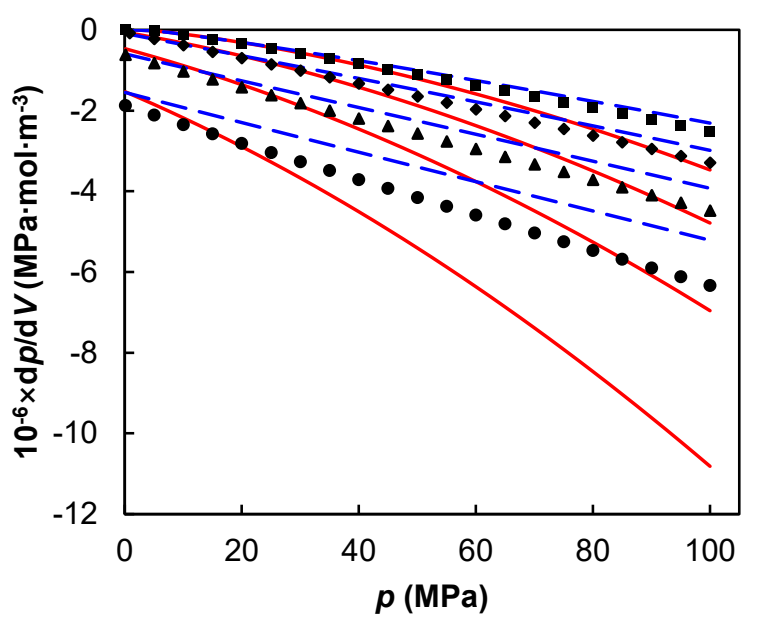

(a)

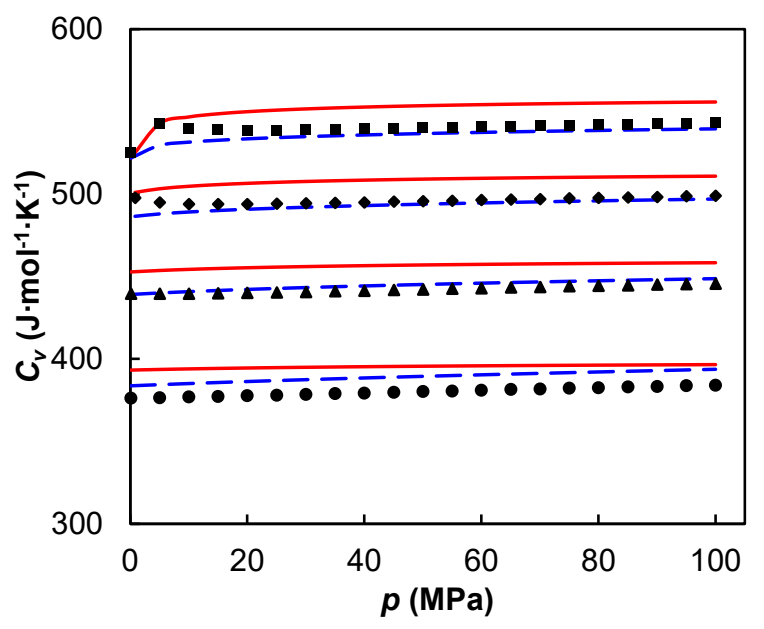

(c)

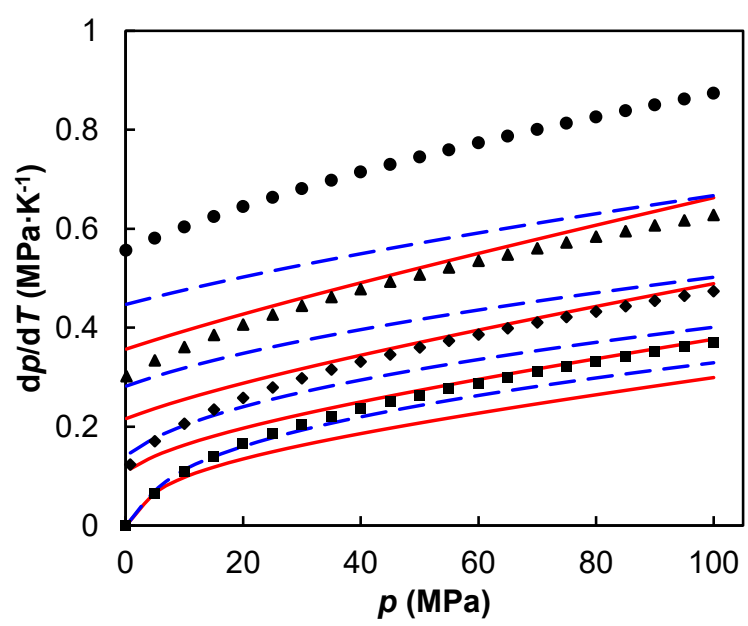

(b)

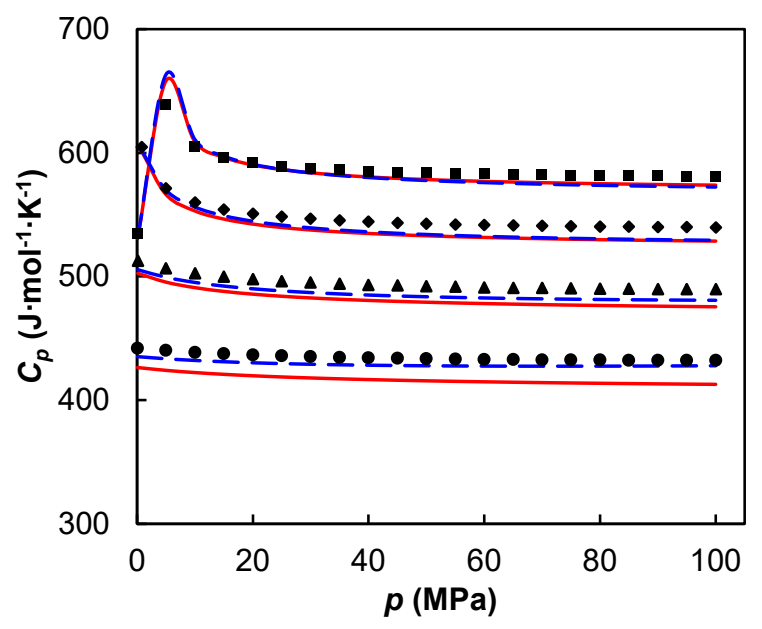

(d)

608 Figure 2. Derivative properties of dodecane with CPA and PC-SAFT: (a) $\mathrm{d} p / \mathrm{d} V$, (b) $\mathrm{d} p / \mathrm{d} T$,

609 (c) $C_{v}$, (d) $C_{p}$. Symbols: ${ }^{58}(\bullet) 400 \mathrm{~K},(\Delta) 500 \mathrm{~K},(\bullet) 600 \mathrm{~K},(\bullet) 700 \mathrm{~K}$; (一) CPA (red), (610 -) PC-SAFT (blue). 


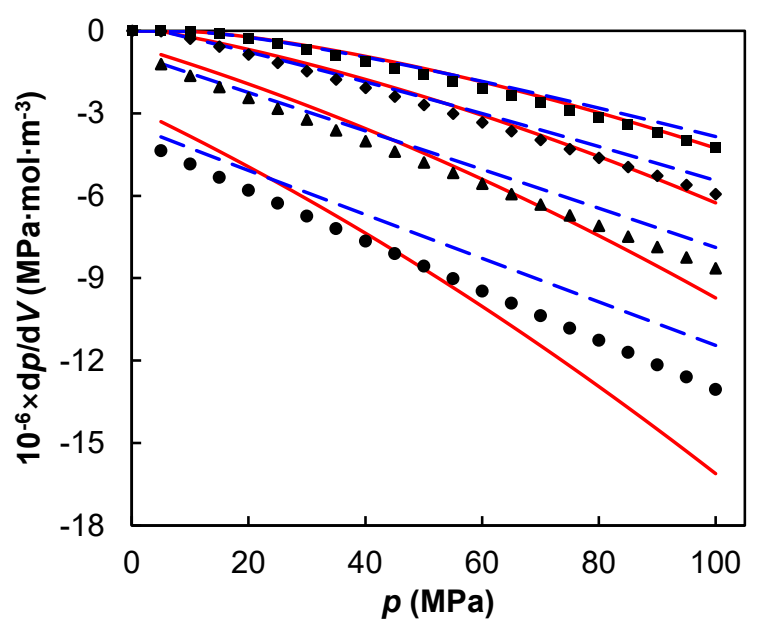

(a)

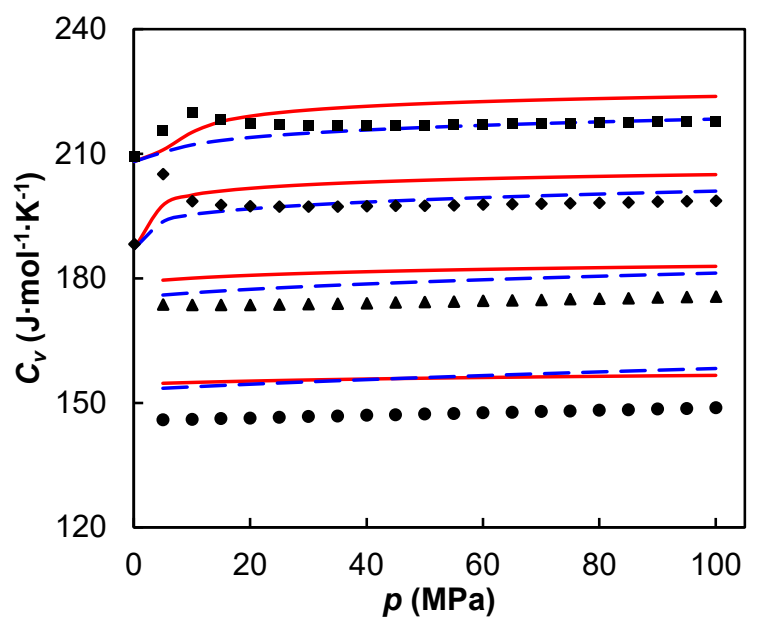

(c)

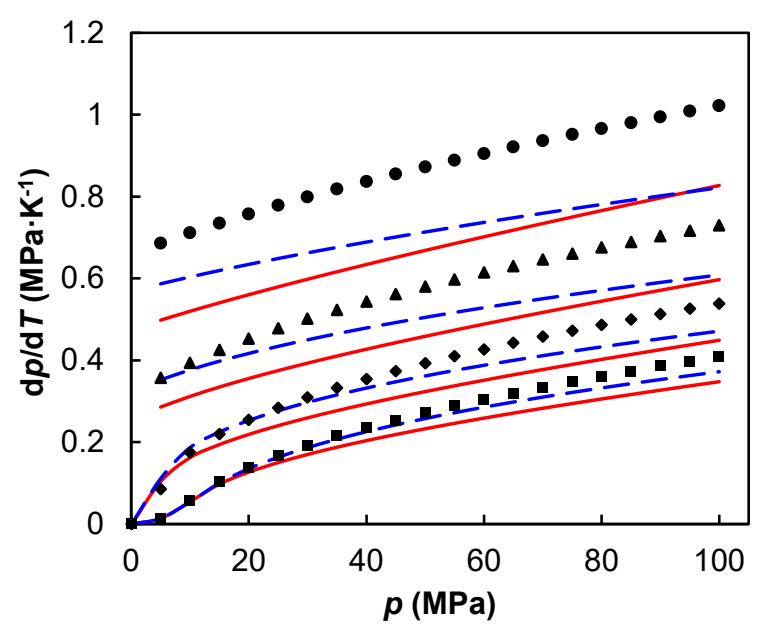

(b)

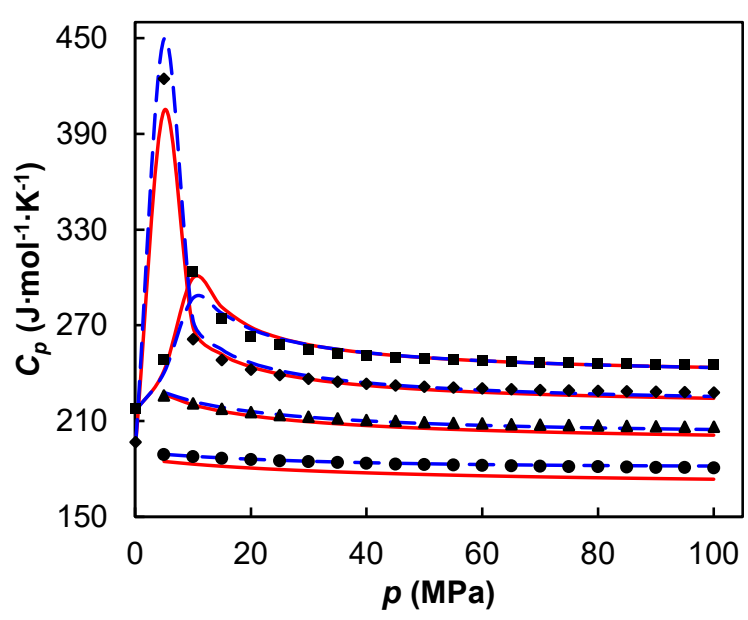

(d)

611 Figure 3. Derivative properties of toluene with CPA and PC-SAFT: (a) $\mathrm{d} p / \mathrm{d} V$, (b) $\mathrm{d} p / \mathrm{d} T$,

612 (c) $C_{v}$, (d) $C_{p}$. Symbols: ${ }^{58}(\bullet) 400 \mathrm{~K},(\boldsymbol{\Delta}) 500 \mathrm{~K},(\bullet) 600 \mathrm{~K},(\bullet) 700 \mathrm{~K}$; (一) CPA (red), (613 -) PC-SAFT (blue).

614 The relative deviations (RDs) of heat capacities for pentane with the two EoS are shown 615 in Figures 4 and 5, in which the deviations in liquid and supercritical phases are considered 616 separately. It is obvious that the two EoS can give lower deviations of both $C_{v}$ and $C_{p}$ at 
617 supercritical conditions than at subcritical ones. This is because, as shown in Figure 1, the

618 percentage of $C_{p}$ id $/ C_{p}$ is larger at higher temperatures. Furthermore, the CPA EoS tends to

619 underestimate the liquid $C_{p}$, and deviations increase with the temperature until positive

620 deviations may be obtained in the supercritical regions. On the other hand, the RDs of $C_{v}$

621 show that this property is typically overestimated by the two EoS for liquid pentane, and

622 the deviations reduce with increasing temperature. Finally, it should be emphasized that,

623 although the above results summarized in Figures 4 and 5 are for pentane, they are actually

624 almost general for most compounds studied in this work.

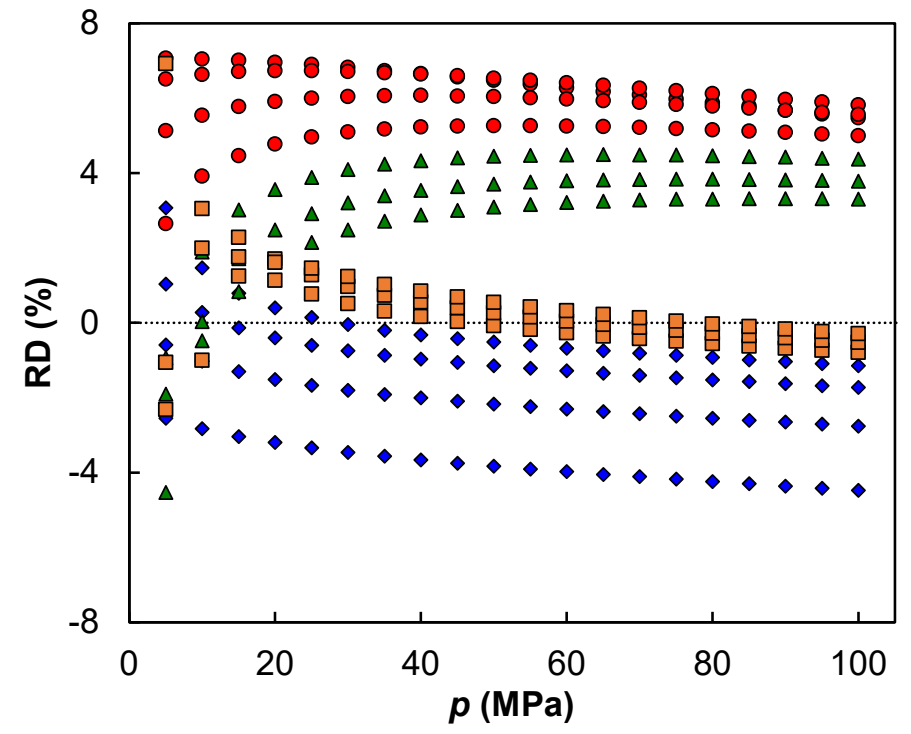

626 Figure 4. Relative deviations of heat capacities with CPA for liquid pentane: $(\bullet) C_{v}(\mathrm{red})$,

$627(\diamond) C_{p}$ (blue) and supercritical pentane: $(\Delta) C_{v}$ (green), (घ) $C_{p}$ (orange). 
628

629

630

631

632

633

634

635

636 can be realized that the accuracy of $C_{p}$ is determined by $C_{v}, \mathrm{~d} p / \mathrm{d} T$, and $\mathrm{d} p / \mathrm{d} V$. Therefore,

637 in some cases, the performance for $C_{v}, \mathrm{~d} p / \mathrm{d} T$, and $\mathrm{d} p / \mathrm{d} V$ may not be satisfactory, but the

638 high accuracy of $C_{p}$ can still be obtained. For example, the AAD of $C_{v}, \mathrm{~d} p / \mathrm{d} T$, and $\mathrm{d} p / \mathrm{d} V$

639 for hydrogen sulfide with CPA and the three-sites association scheme is higher than with

640 PC-SAFT, but the CPA provides a better description of $C_{p}$. This is because the deviations

641 of three properties $\left(C_{v}, \mathrm{~d} p / \mathrm{d} T\right.$, and $\left.\mathrm{d} p / \mathrm{d} V\right)$ are somewhat counterbalanced, leading to a 
642 lower AAD of $1.4 \%$ with CPA compared to an AAD of $3.5 \%$ with PC-SAFT. In addition,

643 the equations of state with different association schemes proposed by Huang and Radosz ${ }^{9}$

644 are also considered in Table 5. As we have mentioned before, a more rigorous scheme (in

645 the terminology used by Huang and Radosz) may not always give better performance for

646 the heat capacities. For example, in Table 5, although the accuracy of $C_{v}$ for water with

647 both CPA and PC-SAFT coupled with the 4C scheme is higher than that with the 3B scheme,

648 the performance for $C_{p}$ with the more rigorous scheme for water (4C) deteriorates.

649 Specially, hydrogen sulfide is considered as both inert compound and associating

650 compound with three different schemes. Although less parameters are included, both

651 models without considering the association still can give good performance for heat

652 capacities, with the AAD of $3 \%$ or so, and this is consistent with the results in ref. 32.

653 Furthermore, among the eight scenarios, CPA without the association shows the lowest

$654 \mathrm{AAD}$, which is $2.8 \%$, for $C_{v}$. Figure 6 shows the derivative properties of water with two

655 EoS at $400 \mathrm{~K}$. From Figures $6 \mathrm{a}$ and $6 \mathrm{~b}$, we can observe that the calculated $\mathrm{d} p / \mathrm{d} V$ and $\mathrm{d} p / \mathrm{d} T$

656 with the $3 \mathrm{~B}$ scheme show the largest deviations from the NIST values among the three

657 schemes, and such deviations decrease significantly when the $4 \mathrm{C}$ scheme is used. This

658 result is also shown in Table 5, in which the AAD of $\mathrm{d} p / \mathrm{d} T$ for water with PC-SAFT and

659 3B scheme is even higher than $96 \%$, while the AAD with $4 \mathrm{C}$ scheme can reduce to $23 \%$.

660 Furthermore, in Figures $6 a$ and $6 b$, each model with different association schemes gives

661 the same slope of $\mathrm{d} p / \mathrm{d} V$ or $\mathrm{d} p / \mathrm{d} T$ curves. The PC-SAFT EoS can give better description of

662 the slopes compared to the results from NIST than CPA. As for the heat capacities, the CPA 
663 EoS with the $4 \mathrm{C}$ and $2 \mathrm{~B}$ schemes respectively describes the $C_{v}$ and $C_{p}$ data best among the 664 six calculation scenarios. Surprisingly, the PC-SAFT EoS with 4C scheme just slightly 665 improves the results of $C_{v}$ compared with the EoS with 3B scheme, and even has a worse 666 performance for $C_{p}$. It should be emphasized that the performance of CPA with the 4C 667 scheme for heat capacities seems quite satisfactory in Figure 6 at $T=400 \mathrm{~K}$, but the AAD 668 of $C_{v}$ and $C_{p}$ for water over a wider temperature range are relatively large, reaching to $5.4 \%$ 669 and $7.9 \%$, respectively.

670 Table 5. AAD of heat capacities for associating compounds with CPA and PC-SAFT

\begin{tabular}{|c|c|c|c|c|c|c|c|c|c|c|c|c|}
\hline \multirow{2}{*}{ Compound } & \multirow{2}{*}{$T / \mathrm{K}$} & \multirow{2}{*}{$p / \mathrm{MPa}$} & \multirow{2}{*}{ Scheme } & \multicolumn{4}{|c|}{$\mathrm{CPA} / \%$} & \multicolumn{4}{|c|}{ PC-SAFT/\% } & \multirow{2}{*}{$\begin{array}{l}\text { No. of } \\
\text { points }\end{array}$} \\
\hline & & & & $C_{p}$ & $C_{v}$ & $\mathrm{~d} p / \mathrm{d} V$ & $\mathrm{~d} p / \mathrm{d} T$ & $C_{p}$ & $C_{v}$ & $\mathrm{~d} p / \mathrm{d} V$ & $\mathrm{~d} p / \mathrm{d} T$ & \\
\hline \multirow[t]{3}{*}{ Water } & $300-700$ & $0.1-100$ & $2 \mathrm{~B}$ & 3.41 & 10.3 & 38.6 & 72.8 & 11.9 & 12.2 & 49.2 & 49.4 & 189 \\
\hline & & & $3 \mathrm{~B}$ & 4.97 & 12.6 & 46.3 & 80.5 & 8.11 & 15.3 & 82.6 & 96.8 & 189 \\
\hline & & & $4 \mathrm{C}$ & 5.37 & 7.92 & 12.8 & 28.4 & 9.68 & 11.4 & 9.08 & 22.9 & 189 \\
\hline \multirow[t]{4}{*}{ Hydrogen sulfide } & $200-500$ & $0.1-100$ & & 3.07 & 2.82 & 9.41 & 5.68 & 3.14 & 2.98 & 5.08 & 1.72 & 132 \\
\hline & & & $1 \mathrm{~A}$ & 3.37 & 17.1 & 7.03 & 14.2 & 4.09 & 5.73 & 4.60 & 1.76 & 132 \\
\hline & & & $2 \mathrm{~B}$ & 0.97 & 13.3 & 5.25 & 11.6 & 2.59 & 2.96 & 4.69 & 1.62 & 132 \\
\hline & & & 3B & 1.40 & 8.81 & 5.19 & 6.92 & 3.45 & 4.21 & 4.55 & 1.86 & 132 \\
\hline \multirow[t]{2}{*}{ Ammonia } & $250-550$ & $0.1-100$ & $3 \mathrm{~B}$ & 2.59 & 5.21 & 6.56 & 6.84 & 5.68 & 10.1 & 5.74 & 4.14 & 147 \\
\hline & & & $4 B$ & 2.39 & 5.82 & 6.73 & 7.68 & 5.65 & 12.6 & 5.40 & 5.22 & 147 \\
\hline \multirow[t]{2}{*}{ Methanol } & $300-600$ & $0.1-100$ & $2 \mathrm{~B}$ & 7.83 & 5.60 & 6.15 & 11.0 & 12.2 & 12.5 & 8.92 & 12.2 & 147 \\
\hline & & & 3B & 5.24 & 8.07 & 18.2 & 14.5 & 4.61 & 17.9 & 17.7 & 29.2 & 147 \\
\hline Overall $^{\mathrm{a}}$ & & & & 3.77 & 7.64 & 11.0 & 15.5 & 6.17 & 11.7 & 9.28 & 15.7 & \\
\hline
\end{tabular}

$671{ }^{a}$ Only the most rigorous schemes (bold) for each compound are included in the overall 672 AAD. 


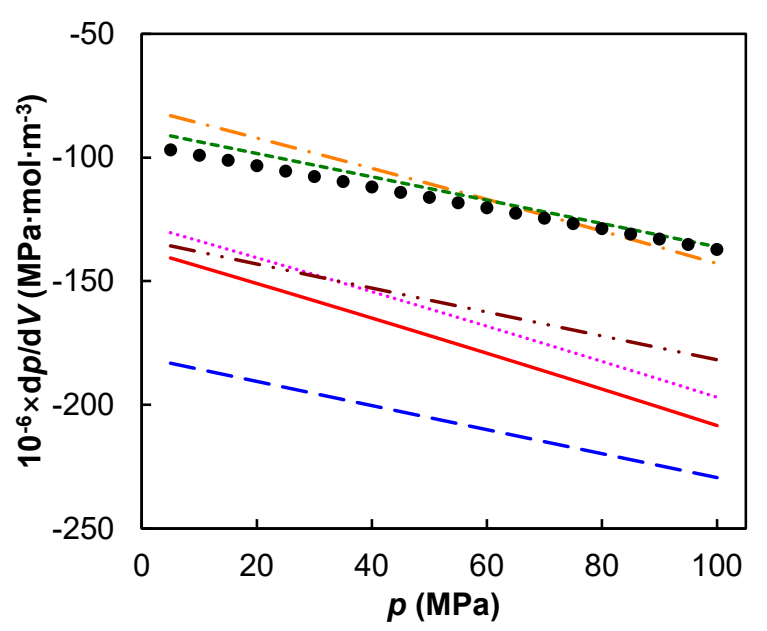

(a)

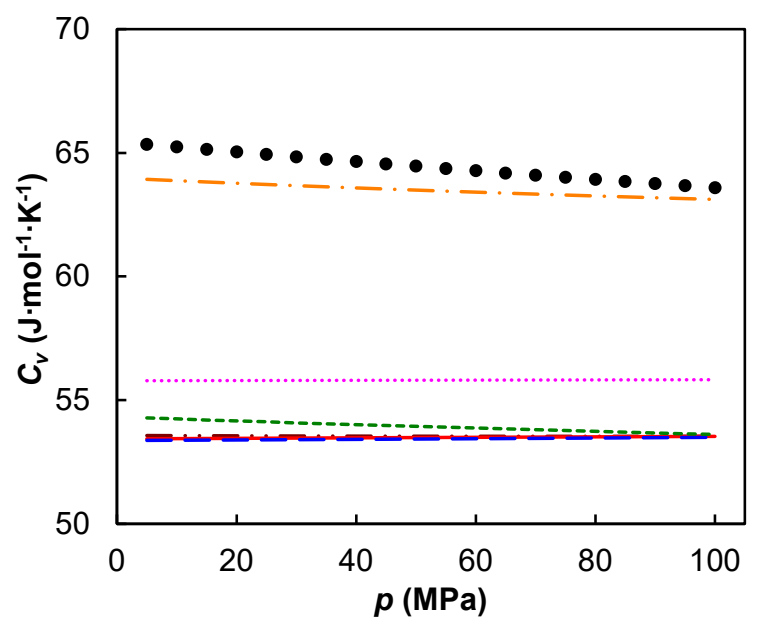

(c)

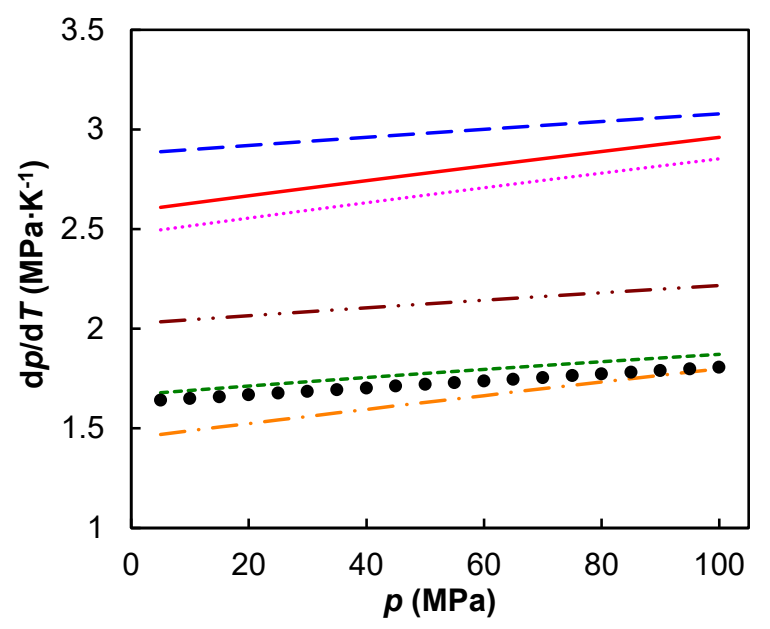

(b)

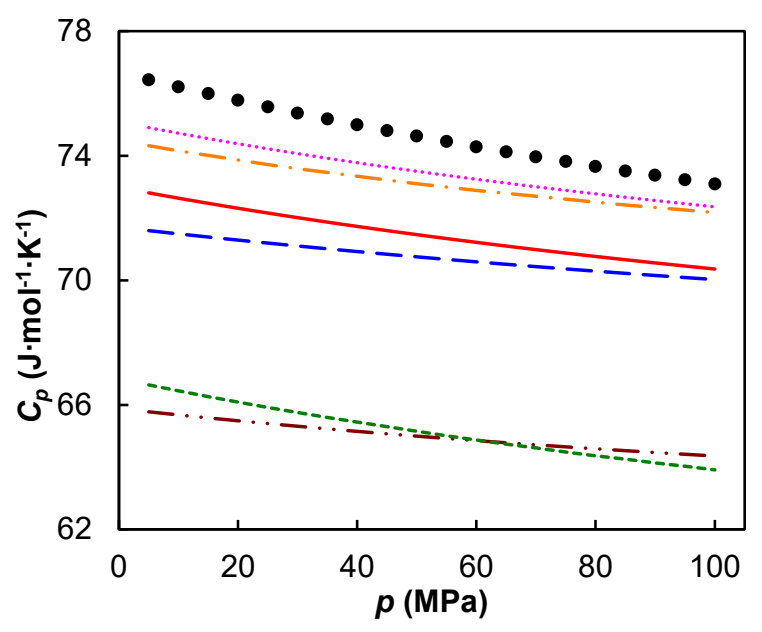

(d)

673 Figure 6. Derivative properties of water with CPA and PC-SAFT at $T=400 \mathrm{~K}$ : (a) $\mathrm{d} p / \mathrm{d} V$,

674 (b) $\mathrm{d} p / \mathrm{d} T$, (c) $C_{v}$, (d) $C_{p} .(\bullet)$ NIST values, ${ }^{58}(\cdots)$ CPA-2B (pink), (-.*-) PC-SAFT-2B

675 (brown), (-) CPA-3B (red), (--) PC-SAFT-3B (blue), (---) CPA-4C (orange), (---) PC-

676 SAFT-4C (green).

677 In the previous section, it is mentioned that some EoS cannot give good performance for 678 both the heat capacity and speed of sound. As seen from the results of Table 5, PC-SAFT 
679 cannot always provide good descriptions of both $C_{v}$ and $C_{p}$. To analyze this further, we 680 have considered the case of ammonia. Table 6 provides six sets of parameters for PC-SAFT 681 for ammonia, and Table 7 gives the results of VLE and derivative properties using these 682 parameter sets, which were fitted to the vapor pressure and saturated liquid density data at $683 T_{r}=0.5-0.9$ in this work. The calculated VLE and derivative properties are compared with 684 the values from DIPPR ${ }^{61}$ and NIST ${ }^{58}$, respectively. The results show that, with sets $1-3$ (in 685 which the association volume $\kappa^{\mathrm{AB}}$ increases) with $3 \mathrm{~B}$ scheme, the AAD of VLE and $C_{p}$ 686 decrease, while the AAD of $C_{v}$ increases. Similar results can also be observed when the 4B 687 scheme is used. This indicates that no matter which association scheme and parameter set 688 are adopted, the PC-SAFT EoS cannot give both good descriptions of both $C_{v}$ and $C_{p}$ for 689 ammonia.

Table 6. PC-SAFT parameters for ammonia

\begin{tabular}{ccccccc}
\hline Set & Scheme & $m$ & $\sigma(\AA)$ & $\varepsilon / k(\mathrm{~K})$ & $\varepsilon^{\mathrm{AB}} / k(\mathrm{~K})$ & $\kappa^{\mathrm{AB}}$ \\
\hline 1 & $3 \mathrm{~B}$ & 2.8587 & 2.1923 & 124.04 & 1084.5 & 0.4435 \\
2 & & 3.3790 & 2.0738 & 123.62 & 835.2 & 0.9133 \\
3 & & 3.8627 & 1.9840 & 123.65 & 580.1 & 1.9543 \\
4 & $4 \mathrm{~B}$ & 3.2723 & 2.0906 & 120.93 & 933.6 & 0.4567 \\
5 & & 3.7231 & 2.0044 & 121.23 & 706.1 & 0.8810 \\
6 & & 4.0257 & 1.9538 & 121.47 & 536.9 & 1.4935 \\
\hline
\end{tabular}

$$
T=200-500 \mathrm{~K} \text { and } p=0.1-100 \mathrm{MPa}
$$

\begin{tabular}{cccccccc}
\hline \multirow{2}{*}{ Set } & \multirow{2}{*}{ Scheme } & \multicolumn{6}{c}{$\mathrm{AAD} / \%$} \\
\cline { 3 - 8 } & & $p_{\text {sat }}$ & $\rho_{\text {sat, } 1}$ & $C_{p}$ & $C_{v}$ & $\mathrm{~d} p / \mathrm{d} V$ & $\mathrm{~d} p / \mathrm{d} T$ \\
\hline 1 & $3 \mathrm{~B}$ & 1.04 & 0.22 & 8.18 & 5.90 & 9.83 & 6.97
\end{tabular}




\begin{tabular}{|c|c|c|c|c|c|c|c|}
\hline \multirow{2}{*}{ Set } & \multirow{2}{*}{ Scheme } & \multicolumn{6}{|c|}{$\mathrm{AAD} / \%$} \\
\hline & & $p_{\text {sat }}$ & $\rho_{\mathrm{sat}, 1}$ & $C_{p}$ & $C_{v}$ & $\mathrm{~d} p / \mathrm{d} V$ & $\mathrm{~d} p / \mathrm{d} T$ \\
\hline 2 & & 0.41 & 0.14 & 5.68 & 10.12 & 5.74 & 4.14 \\
\hline 3 & & 0.05 & 0.09 & 4.32 & 13.96 & 6.28 & 8.02 \\
\hline 4 & $4 B$ & 0.72 & 0.11 & 7.63 & 9.04 & 8.10 & 5.22 \\
\hline 5 & & 0.24 & 0.08 & 5.65 & 12.60 & 5.40 & 5.22 \\
\hline 6 & & 0.04 & 0.06 & 4.68 & 14.99 & 6.68 & 8.85 \\
\hline
\end{tabular}

693 In addition to methanol presented in Table 5, the isobaric heat capacities of other alcohols

694 were also calculated with CPA and PC-SAFT. The deviations of the calculated $C_{p}$ from the 695 experimental data ${ }^{65-80}$ are shown in Table 8 . Two association schemes (2B and $\left.3 \mathrm{~B}\right)$ are 696 considered with the two EoS. The results show that the different schemes with CPA may 697 affect the $C_{p}$ calculation for each compound, but the effect on the overall performance is 698 small. Compared to CPA, PC-SAFT provides a slightly lower AAD with 3B scheme (5.0\%) 699 and almost an identical AAD with 2B scheme (5.9\%). Therefore, we can conclude that, the 700 two EoS with two different association schemes have the similar overall accuracy of $C_{p}$ for 701 alcohols. Figure 7 shows the relative deviations of $C_{p}$ with the two EoS and two schemes 702 for pentanol at atmospheric pressure. We can see that all four scenarios have almost 703 identical and low deviations in the gas region, with an average deviation about $2 \%$, and the 704 RDs in the liquid region show similar distributions. This conclusion can also be given from 705 Figure 8, in which the calculated $C_{p}$ for 2-butanol is compared with the experimental data 706 from refs 68 and 78 at $p=0.1 \mathrm{MPa}$. The identical $C_{p}$ results at the gas state can be observed 707 in Figure 8, since the four curves nearly overlap in this region. 
Table 8. AAD of $C_{p}$ for alcohols with CPA and PC-SAFT

\begin{tabular}{ccccccccc}
\hline \multirow{2}{*}{ Compound } & \multirow{2}{*}{$T / \mathrm{K}$} & $p / \mathrm{MPa}$ & \multicolumn{2}{c}{$\mathrm{CPA} / \%$} & \multicolumn{2}{c}{ PC-SAFT/\% } & \multirow{2}{*}{ No. of } & \multirow{2}{*}{ Ref. } \\
\cline { 5 - 7 } & & & $2 \mathrm{~B}$ & $3 \mathrm{~B}$ & $2 \mathrm{~B}$ & $3 \mathrm{~B}$ & Points & \\
\hline Ethanol & $265-591$ & $0.1-25.1$ & 11.3 & 11.9 & 1.90 & 4.09 & 80 & $65-68$ \\
Propanol & $154-603$ & 0.1 & 6.12 & 6.18 & 6.36 & 6.34 & 64 & 68,69 \\
Butanol & $188-603$ & $0.1-50$ & 4.59 & 5.09 & 6.09 & 7.11 & 119 & $68,70-72$ \\
Pentanol & $200-574$ & 0.1 & 4.19 & 4.12 & 8.12 & 3.43 & 65 & 68,69 \\
Hexanol & $326-571$ & $2-30$ & 3.88 & 1.24 & 1.94 & 1.31 & 66 & 73 \\
Heptanol & $326-571$ & $2-30$ & 4.58 & 1.93 & 6.48 & 3.00 & 68 & 73 \\
Octanol & $326-571$ & $2-30$ & 6.29 & 5.54 & 2.85 & 2.47 & 66 & 73 \\
Nonanol & $281-330$ & 0.1 & 4.54 & 11.0 & 11.2 & 10.9 & 24 & 74 \\
Decanol & $326-571$ & $2-30$ & 2.63 & 3.12 & 2.03 & 3.37 & 68 & 73 \\
2-Propanol & $253-573$ & $0.1-40$ & 7.26 & 8.47 & 8.27 & 8.27 & 167 & $75-77$ \\
2-Butanol & $188-583$ & $0.1-25$ & 10.3 & 8.57 & 14.9 & 10.3 & 84 & $68,78,79$ \\
iso-Butanol & $298-493$ & $0.1-4$ & 5.24 & 4.92 & 7.40 & 3.49 & 97 & 69,77 \\
tert-Butanol & $353-453$ & $1-4$ & 2.46 & 2.35 & 1.25 & 1.36 & 62 & 77 \\
tert-Pentanol & $298-369$ & 0.1 & 1.92 & 1.55 & 1.38 & 1.22 & 73 & 80 \\
Overall & & & 5.67 & 5.57 & 5.90 & 4.99 & & \\
\hline
\end{tabular}

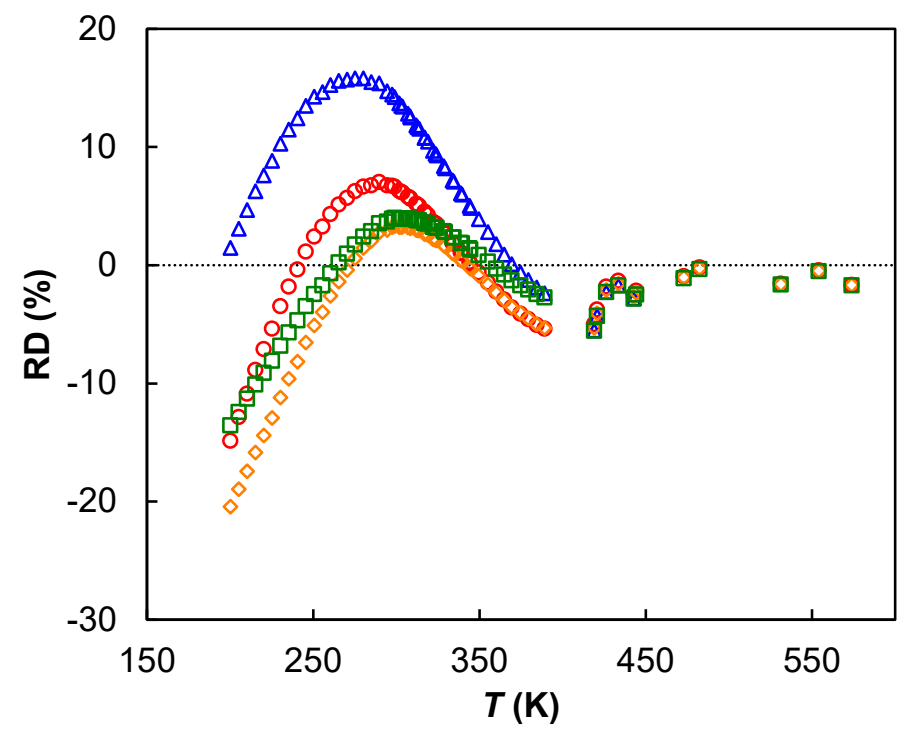

710 Figure 7. Relative deviations of $C_{p}$ with CPA and PC-SAFT for pentanol at $p=0.1 \mathrm{MPa}$ ( $(\circ)$

711 CPA-2B (red), $(\triangle)$ PC-SAFT-2B (blue), $(\diamond)$ CPA-3B (orange), ( $\square$ ) PC-SAFT-3B (green). 


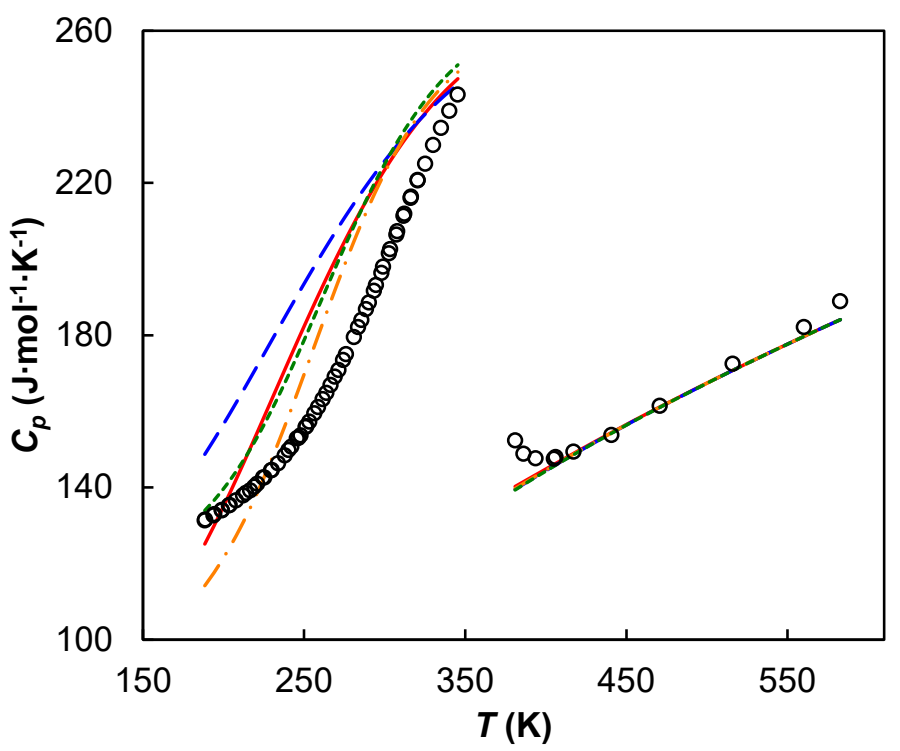

713 Figure 8. $C_{p}$ of 2-butanol with CPA and PC-SAFT at $p=0.1 \mathrm{MPa}:(\circ)$ Experiment ${ }^{68,78},(-)$

714 CPA-2B (red), (--) PC-SAFT-2B (blue), (-•-) CPA-3B (orange), (---) PC-SAFT-3B 715 (green).

716 Figure 9 shows the relative deviations of $C_{p}$ and residual $C_{p}$ with PC-SAFT and the 3B 717 scheme for butanol at atmospheric pressure. In the liquid phase, the RD values of $C_{p}$ are 718 almost half of the RD of $C_{p}{ }^{\text {res }}$, while the differences between the RD values of $C_{p}$ and $C_{p}{ }^{\text {res }}$ 719 become much larger for the gaseous butanol. This is because the percentage of $C_{p}$ res in $C_{p}$ 720 is about $50 \%$ for the liquid butanol, while this value decrease to lower than $10 \%$ in the gas 721 phase. The similar results can also be observed for water in Figure 1. In fact, as the 722 contribution of the association interaction to $C_{p}$ is noticeable in the liquid phase, the ratio 723 of $C_{p}$ id $/ C_{p}$ for associating fluids is generally lower than that for non-associating substances, 724 while it becomes much higher in the gaseous region with the break of hydrogen bond, 725 leading to a dominant contribution from ideal gas $C_{p}$. This can also explain why CPA and 
PC-SAFT with both 2B and 3B schemes show the same and good performance on $C_{p}$ at the gas condition in Figures 7 and 8.

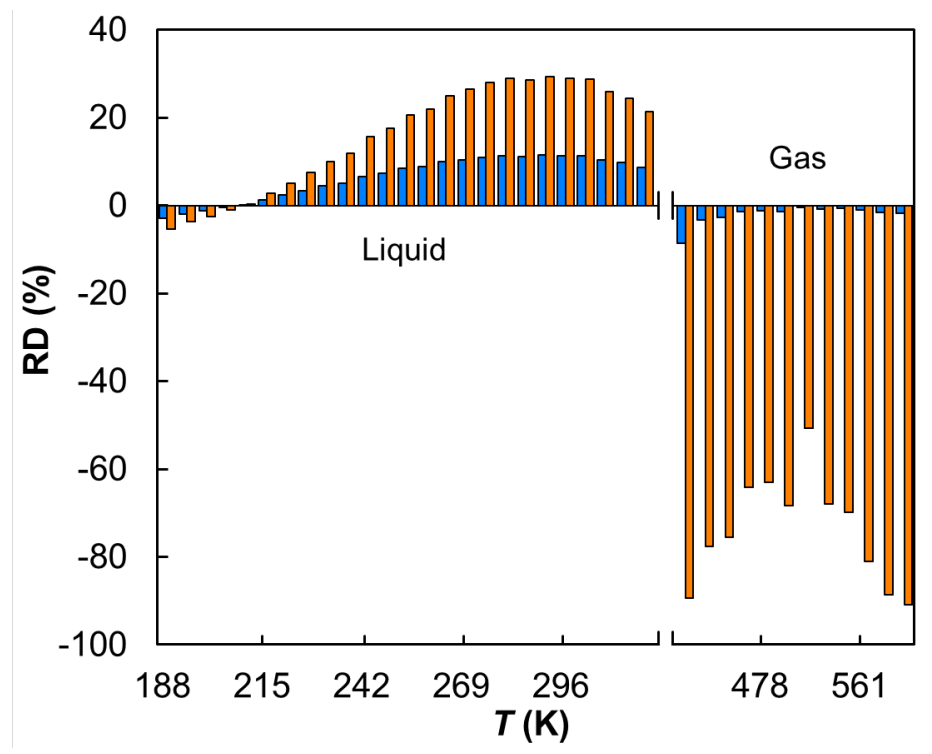

729 Figure 9. Relative deviations of $C_{p}$ and $C_{p}{ }^{\text {res }}$ with PC-SAFT and 3B scheme for butanol at $730 p=0.1 \mathrm{MPa}(\boldsymbol{\square}) C_{p}$ (blue), (घ) $C_{p}^{\text {res }}$ (orange).

\subsection{Critical Isotherms}

732 The derivative properties at reduced temperature $T_{\mathrm{r}}=T / T_{\mathrm{c}}=1$ and pressures up to $2 p_{\mathrm{c}}$ are 733 also estimated with CPA and PC-SAFT in this work, and the AAD compared to the NIST 734 values $^{58}$ are shown in Table 9. It should be noted that, for a fair comparison, the above 735 critical temperature and pressure for NIST values are from experiments (the "real" $T_{\mathrm{c}}$ and $736 p_{\mathrm{c}}$ ), while the critical properties for each model are calculated by the model itself. Only 737 some of previously studied compounds from Tables 4 and 5 are considered, as not all the correlations from NIST can be used for fluids near the critical points. From Table 9, it is obvious that CPA is somewhat more accurate on the estimation of heat capacities at critical 
740 isotherms with the overall AAD of $6.7 \%$ (compared to $7.8 \%$ for PC-SAFT), while PC-

741 SAFT gives lower deviations of $\mathrm{d} p / \mathrm{d} V$ and $\mathrm{d} p / \mathrm{d} T$ with the overall AAD of $24 \%$ and $7.2 \%$,

742 respectively. The calculated results for dodecane and toluene are plotted in Figures 10 and

74311 , respectively, in which the $\mathrm{d} p / \mathrm{d} V$ is given as $\ln (-\mathrm{d} p / \mathrm{d} V)$ for a clear illustration at the gas

744 condition. On the basis of Figures 10 and 11, the following conclusions can be obtained:

745 First, compared with CPA, there is a superiority of PC-SAFT in $\mathrm{d} p / \mathrm{d} V$ and $\mathrm{d} p / \mathrm{d} T$ at the

746 liquid state. Second, neither of the two EoS can capture the $C_{v}$ divergence at the critical

747 point. Third, the performance of the two EoS for $C_{p}$ is quite similar at $T_{\mathrm{r}}=1$, and both models

748 give rather satisfactory descriptions outside of the critical regions, while the deviations

749 become much larger in the vicinity of the critical points.

Table 9. AAD of heat capacities with CPA and PC-SAFT at $T_{\mathrm{r}}=1$

\begin{tabular}{|c|c|c|c|c|c|c|c|c|c|c|}
\hline \multirow{2}{*}{ Compound } & \multirow{2}{*}{ Scheme } & \multicolumn{4}{|c|}{ CPA $/ \%$} & \multicolumn{4}{|c|}{ PC-SAFT $/ \%$} & \multirow{2}{*}{$\begin{array}{l}\text { No. of } \\
\text { points }\end{array}$} \\
\hline & & $C_{p}$ & $C_{v}$ & $\mathrm{~d} p / \mathrm{d} V$ & $\mathrm{~d} p / \mathrm{d} T$ & $C_{p}$ & $C_{v}$ & $\mathrm{~d} p / \mathrm{d} V$ & $\mathrm{~d} p / \mathrm{d} T$ & \\
\hline Methane & & 6.89 & 5.90 & 20.5 & 8.11 & 11.4 & 4.90 & 14.7 & 3.05 & 91 \\
\hline Butane & & 4.80 & 2.35 & 28.1 & 9.41 & 5.82 & 1.75 & 19.7 & 3.38 & 75 \\
\hline Dodecane & & 4.56 & 0.97 & 38.4 & 9.74 & 4.57 & 1.56 & 33.1 & 14.4 & 36 \\
\hline Isobutane & & 5.83 & 2.38 & 26.4 & 8.89 & 6.94 & 2.03 & 19.7 & 3.11 & 73 \\
\hline Toluene & & 6.00 & 1.64 & 28.6 & 7.56 & 6.47 & 2.04 & 22.4 & 8.07 & 82 \\
\hline Carbon dioxide & & 8.59 & 8.81 & 25.6 & 8.12 & 7.20 & 13.3 & 18.2 & 3.92 & 74 \\
\hline Nitrogen & & 7.32 & 6.73 & 20.2 & 7.91 & 11.5 & 5.79 & 14.8 & 3.31 & 67 \\
\hline \multirow[t]{3}{*}{ Water } & $2 \mathrm{~B}$ & 6.95 & 12.0 & 42.4 & 12.8 & 11.9 & 7.96 & 103 & 33.1 & 88 \\
\hline & $3 \mathrm{~B}$ & 8.49 & 13.6 & 49.3 & 16.2 & 16.3 & 20.7 & 108 & 35.5 & 88 \\
\hline & $4 \mathrm{C}$ & 8.07 & 6.16 & 48.5 & 19.3 & 6.73 & 8.74 & 53.4 & 20.3 & 88 \\
\hline Overall $^{\mathrm{a}}$ & & 6.66 & 4.61 & 29.3 & 10.1 & 7.79 & 5.27 & 24.4 & 7.24 & \\
\hline
\end{tabular}

$751{ }^{a}$ Only the 4C scheme for water is included in the overall AADs. 


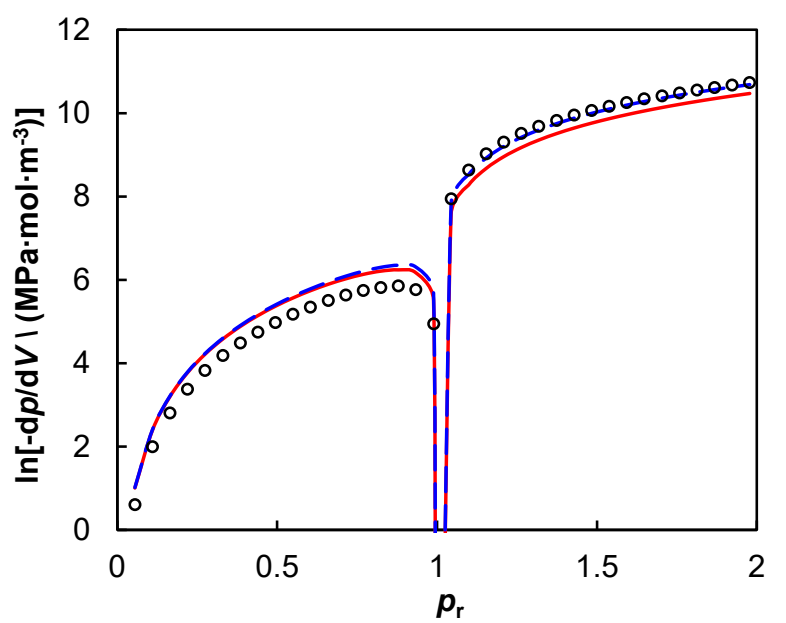

(a)

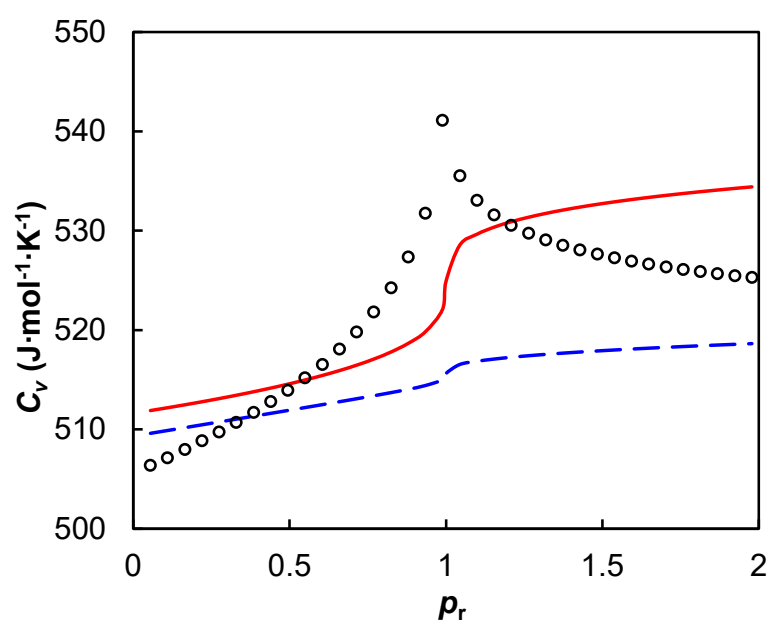

(c)

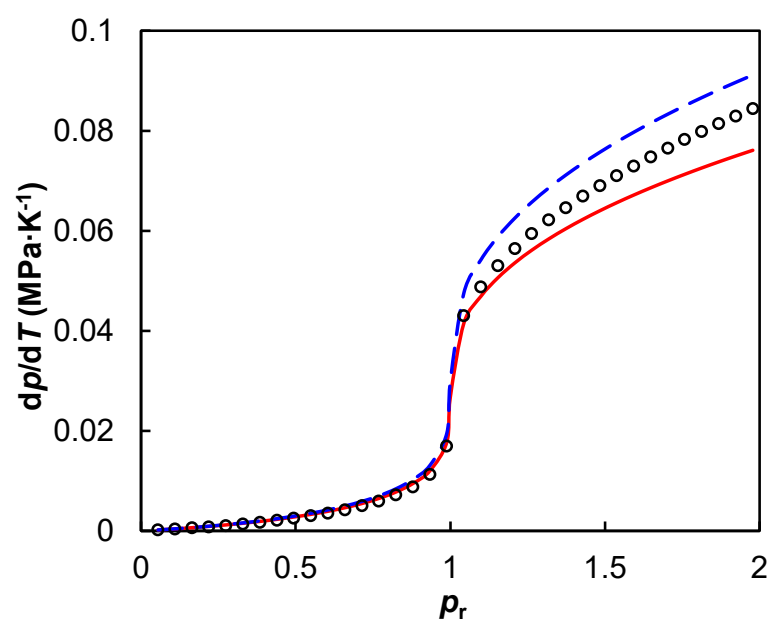

(b)

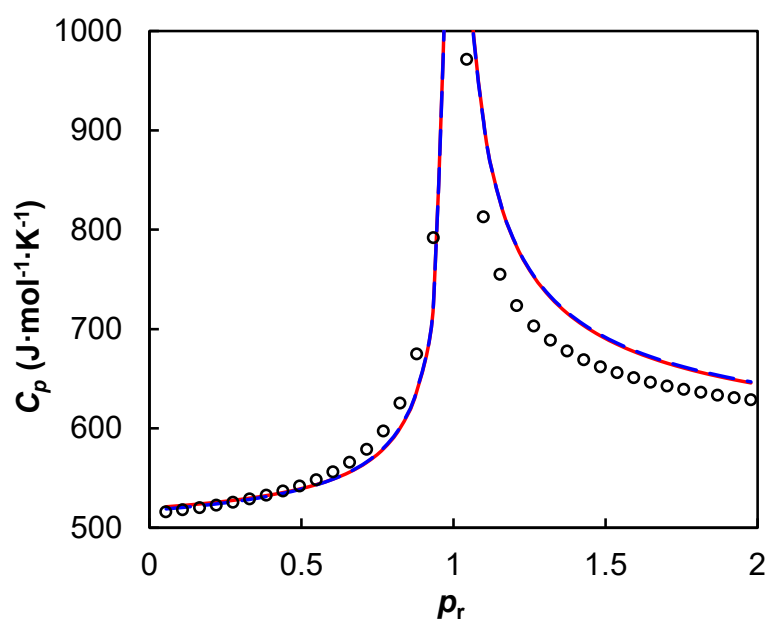

(d)

752 Figure 10. Derivative properties of dodecane with CPA and PC-SAFT at $T_{\mathrm{r}}=1:$ (a) $\mathrm{d} p / \mathrm{d} V$,

753 (b) $\mathrm{d} p / \mathrm{d} T$, (c) $C_{v}$, (d) $C_{p}$. (०) NIST values, ${ }^{58}$ (一) CPA (red), (--) PC-SAFT (blue). 


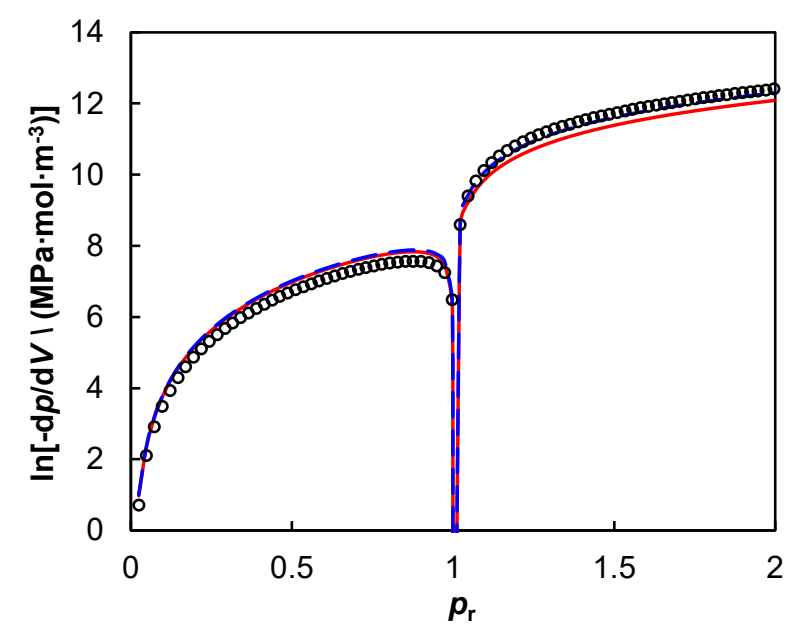

(a)

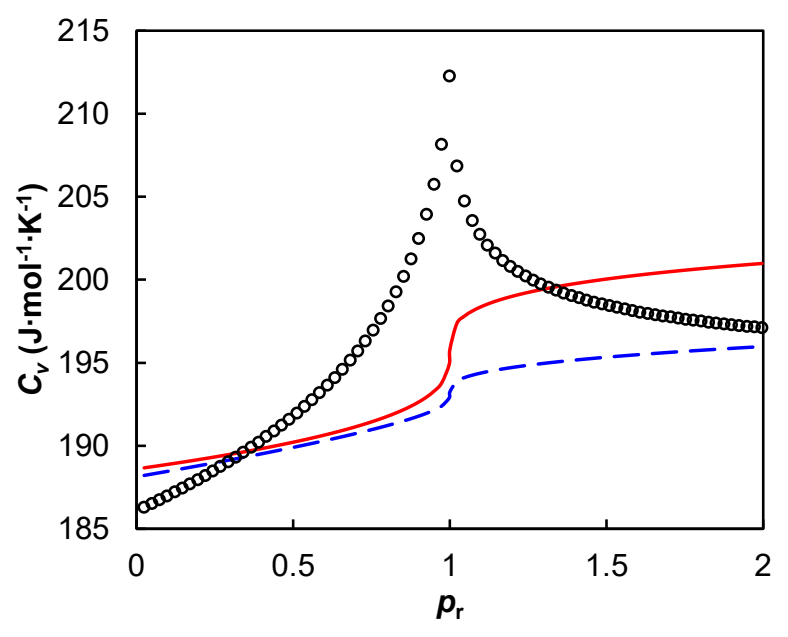

(c)

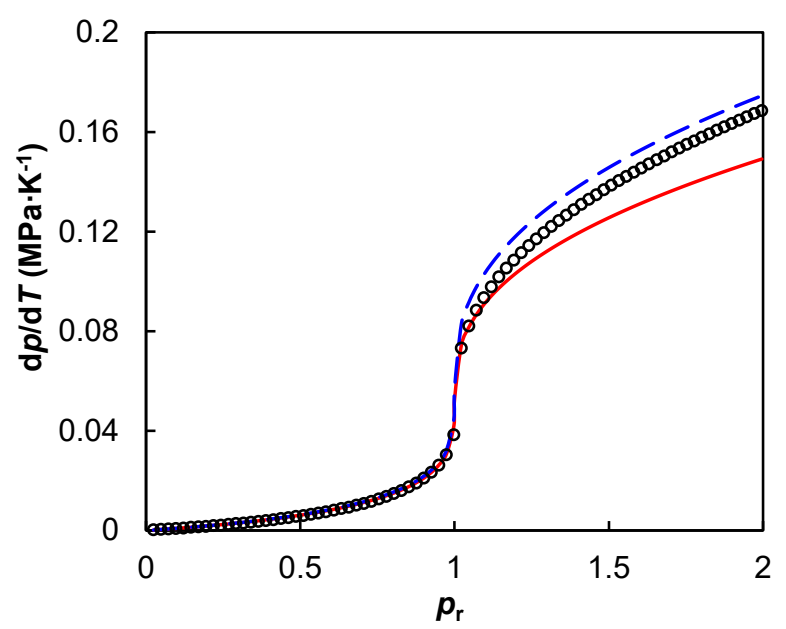

(b)

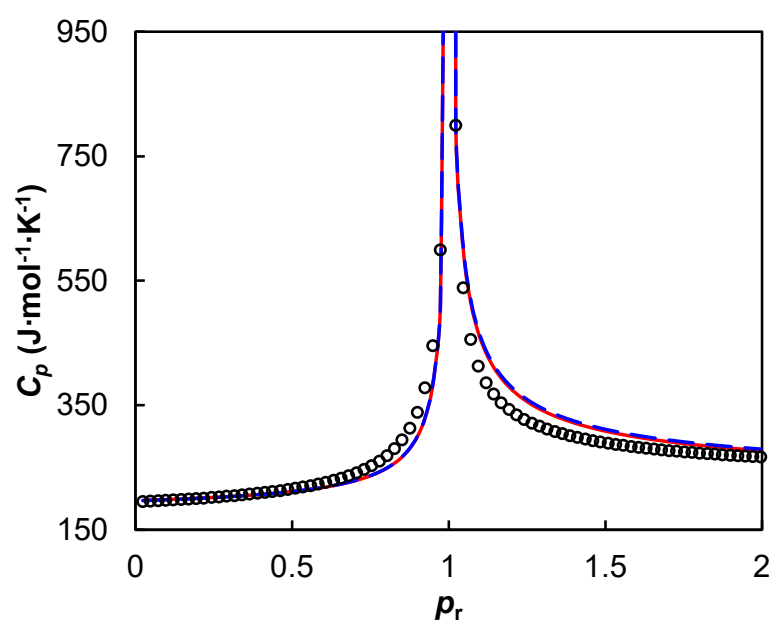

(d)

754 Figure 11. Derivative properties of toluene with CPA and PC-SAFT at $T_{\mathrm{r}}=1:$ (a) $\mathrm{d} p / \mathrm{d} V$, (b)

$755 \mathrm{~d} p / \mathrm{d} T$, (c) $C_{v}$, (d) $C_{p} .\left(\right.$ ( ) NIST values, ${ }^{58}$ (一) CPA (red), (--) PC-SAFT (blue).

756 In Figure 12 is compared the performance of the two EoS with 2B, 3B, and 4C schemes

757 for the heat capacities of water. It is shown that CPA with the 2B scheme and PC-SAFT

758 with the $4 \mathrm{C}$ scheme have the similar good accuracy for $C_{p}$, with the $\mathrm{AAD}$ of both models

759 lower than $7 \%$. The CPA EoS with the $4 \mathrm{C}$ scheme gives the best description of $C_{v}$ among 

can be observed when the $3 \mathrm{~B}$ scheme is considered. Similar to dodecane and toluene, the deviations of $C_{p}$ for water are also quite low at the region far from the critical point, and become much higher when close to the critical region. This is because both CPA and PCSAFT are developed on the basis of the mean-field theory, in which the density fluctuations in the vicinity of critical point are not considered. As for the $C_{v}$, it is encouraging that both EoS with the $4 \mathrm{C}$ scheme can slightly reproduce the $C_{v}$ maxima at their critical points, and this is not observed for other compounds. However, none of the models can successfully describe the $\mathrm{d} p / \mathrm{d} V$ of gaseous water, and the RDs for this property in the gas phase are usually higher than $80 \%$ and even reach to $160 \%$ with PC-SAFT and the $3 \mathrm{~B}$ scheme.

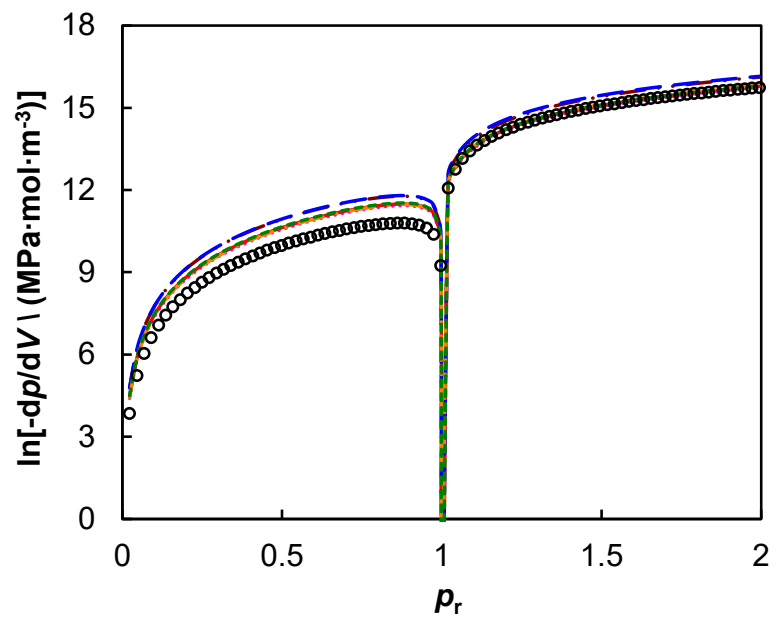

(a)

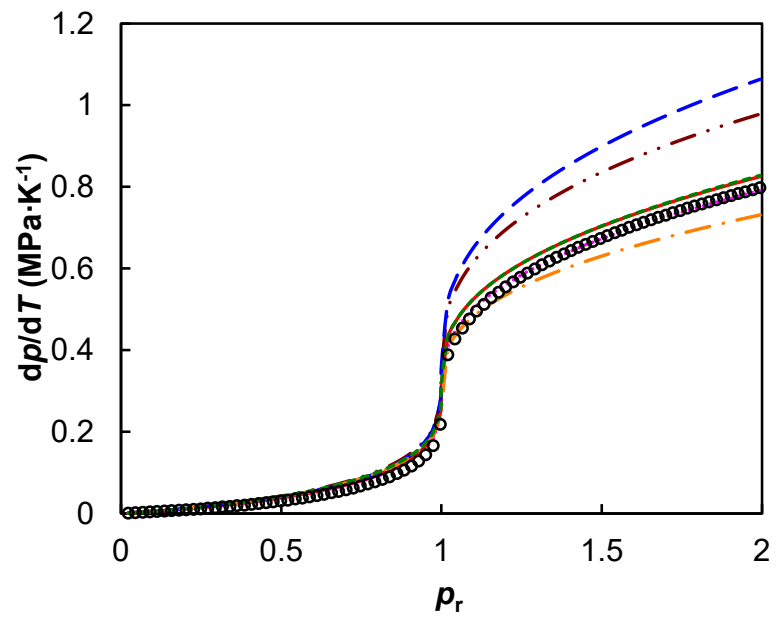

(b) 


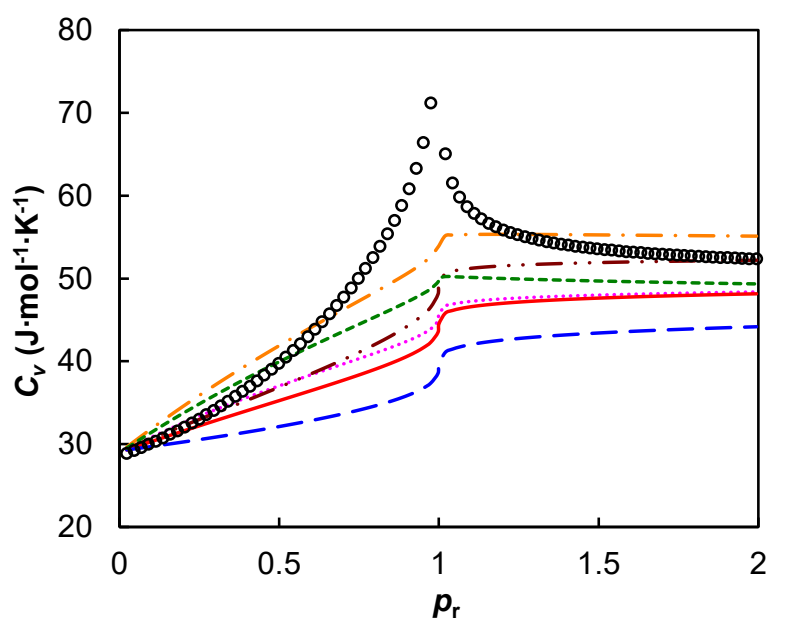

(c)

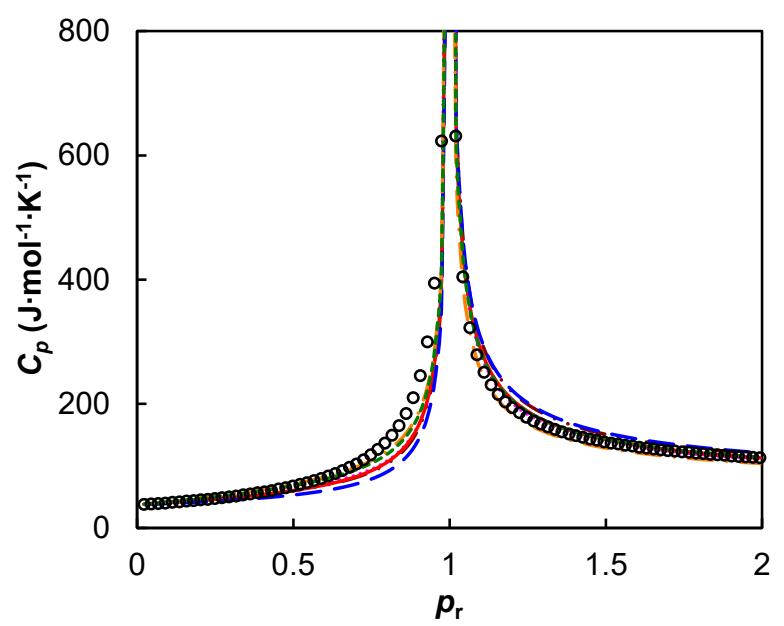

(d)

770 Figure 12. Derivative properties of water with CPA and PC-SAFT at $T_{\mathrm{r}}=1:$ (a) $\mathrm{d} p / \mathrm{d} V$, (b) $\mathrm{d} p / \mathrm{d} T$, (c) $C_{v}$, (d) $C_{p} .(\mathrm{\circ})$ NIST values, ${ }^{58}(\cdots \cdot)$ CPA-2B (pink), (-・-) PC-SAFT-2B (brown),

772 (-) CPA-3B (red), (--) PC-SAFT-3B (blue), (-•-) CPA-4C (orange), (--) PC-SAFT-4C 773 (green).

774 Next, the RD distributions of four properties in eq 2 with CPA for nitrogen are analyzed 775 in Figure 13, from which we can see that the deviations are larger when we are closer to 776 the critical point. Furthermore, we can observe that the RDs of $C_{v}$ and $C_{p}$ show similar 777 trends in the gas phase, especially in the region far away from the critical point. This is due 778 to the significant values of the ideal gas contributions to heat capacities at this condition, 779 and the values are all higher than $70 \%$ at $p_{\mathrm{r}}<0.5$ for both $C_{v}$ and $C_{p}$. In addition, it can also 780 be noted that although the ratio of $C_{p}$ id $/ C_{p}$ is only about $30 \%$ at $p_{\mathrm{r}}>1$, the large deviations 781 of $\mathrm{d} p / \mathrm{d} V$ and $\mathrm{d} p / \mathrm{d} T$ can be somewhat counterbalanced, leading to relatively low deviations 782 for $C_{p}$. This can explain why CPA has worse performance for $\mathrm{d} p / \mathrm{d} V$ and $\mathrm{d} p / \mathrm{d} T$ but still may 


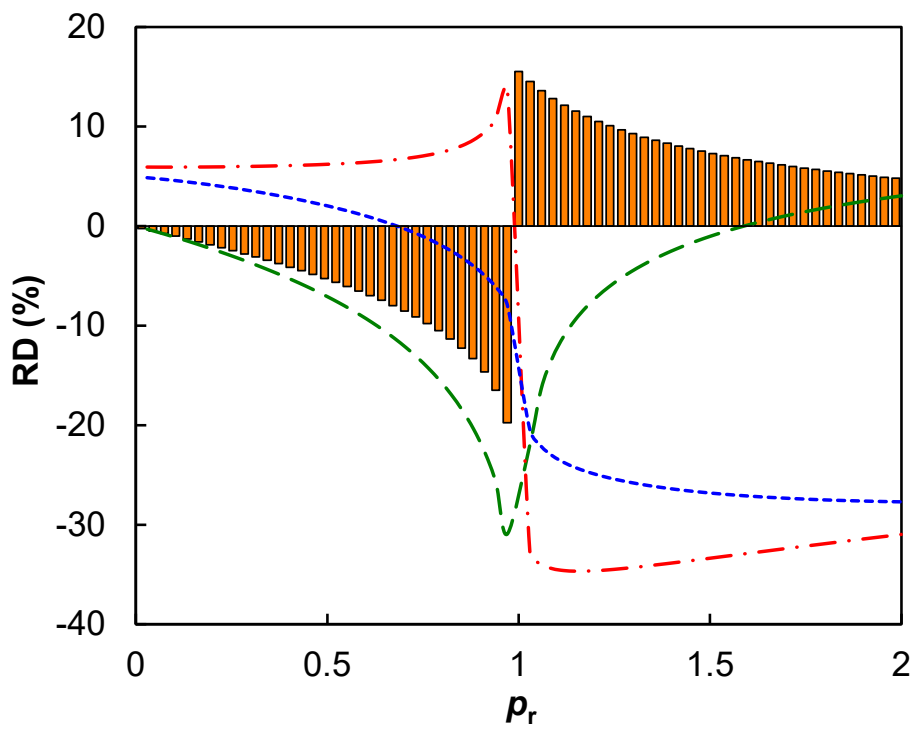

Figure 13. Relative deviations of derivative properties for nitrogen with CPA at $T_{\mathrm{r}}=1:(\square)$ $C_{p},(--) C_{v}$ (green), (-•-) $\mathrm{d} p / \mathrm{d} V($ red $),(--)(\mathrm{d} p / \mathrm{d} T)^{2}$ (blue).

Closing this section, some key points can be summarized. First, for non-associating compounds at the compressed conditions, PC-SAFT shows better performance for the heat capacities than CPA. For associating fluids with the most rigorous schemes (i.e. the scheme with the most associating sites), the two EoS show similar accuracy for the description of $C_{p}$ for alcohols, while CPA gives higher accuracy for other associating fluids, such as water, hydrogen sulfide and ammonia. Second, for the investigated fluids along the critical isotherms, the overall accuracy of heat capacities with CPA is higher than that with PCSAFT, although the latter have lower AAD of $C_{p}$ for carbon dioxide and water. Third, although the most rigorous schemes are considered, the heat capacities may still not be better estimated with EoS compared to when an "approximate" association scheme is used. 
797 Our fourth comment is that, in some cases, the $C_{v}$ and $C_{p}$ cannot be both well represented 798 via an EoS. Fifth, the $C_{v}$ maxima can usually not be captured using EoS. Our final comment 799 is that the ideal gas contributions to heat capacities are particularly high at the gaseous and 800 supercritical conditions far away from the critical point, thus, in these cases, satisfactory results of heat capacities can be obtained if accurate ideal gas heat capacities are available.

\section{Conclusions}

804 In this work we have presented a systematic evaluation of the performance of CPA, PC805 SAFT and other models (mostly SAFT-related) with respect to heat capacities and a few 806 other properties. From the literature studies, the most important conclusion is, except for

PC-SAFT can give the best balance between the performance on heat capacities and speed be a potential tool for the calculation of heat capacities. Furthermore, the performance of soft-SAFT for $C_{p}$ for alcohols is not as good as other EoS.

814 An important observation is about the relative significance of the ideal and residual heat capacity contributions, and we conclude that the ideal gas contribution, which can usually be well estimated, often plays a dominant role in heat capacities at the gas and supercritical conditions far from the critical points. Therefore, in these regions, the heat capacities are 
818 easier to be well described by an EoS, even though the performance for the residual values

819 is unsatisfactory. In terms of the contributions to residual heat capacities, for associating

820 compounds, the contribution of the association term is quite significant, while it becomes

821 much weaker at higher temperatures and/or for longer molecules. For non-associating

822 fluids, the dominant contribution is from the dispersion term. Thus, a higher-order

823 perturbation term for an EoS may be required for a better description of residual heat

824 capacities.

825 Finally, from the calculations performed in this study, we observe that PC-SAFT shows

826 higher accuracy of the heat capacities for non-associating compounds than CPA, while the

827 differences between the two models and different schemes for alcohols are not significant.

828 In addition, the $C_{v}$ at critical isotherms cannot well described by both CPA and PC-SAFT,

829 and the deviations of $C_{p}$ are more significant when we are closer to the critical points.

830 Therefore, we conclude that the performance of CPA and PC-SAFT for residual heat

831 capacities still needs improvement, especially in the region near the critical point.

833 Acknowledgement

834 The support provided by the National Science Fund for Distinguished Young Scholars 835 of China (No. 51525604), the Foundation for Innovative Research Groups of the National 836 Natural Science Foundation of China (No. 51721004), and the China Scholarship Council 837 (No. 201906280322) for the completion of the present work is gratefully acknowledged. 


\section{Supporting Information}

840 Parameters of the CPA and PC-SAFT EoS.

\section{References}

[1] Zábranský, M.; Růžička Jr, V.; Domalski, E. S. Heat capacity of liquids: critical review and recommended values. Supplement I. J. Phys. Chem. Ref. Data 2001, 30, 1199-1689. to ask. J. Solut. Chem. 2010, 39, 1777-1818.

[2] Wilhelm, E. What you always wanted to know about heat capacities, but were afraid

[3] Wilhelm, E. Heat capacities: introduction, concepts and selected applications. In heat capacities. liquids, solutions and vapours; Wilhelm, E., Letcher, T. M., Eds.; RSC Publishing: Cambridge, U.K., 2010.

[4] Segovia, J. J.; Vega-Maza, D.; Chamorro, C. R.; Martín, M. C. High-pressure isobaric heat capacities using a new flow calorimeter. J. Supercrit. Fluids 2008, 46, 258-264. Data 2020, 65, 690-695.

[7] Chapman, W. G.; Gubbins, K. E.; Jackson, G.; Radosz, M. SAFT: Equation-of-state solution model for associating fluids. Fluid Phase Equilib. 1989, 52, 31-38. 
860 [8] Chapman, W. G.; Gubbins, K. E.; Jackson, G.; Radosz, M. New reference equation of 861 state for associating liquids. Ind. Eng. Chem. Res. 1990, 29, 1709-1721.

862 [9] Huang, S. H.; Radosz, M. Equation of state for small, large, polydisperse, and 863 associating molecules. Ind. Eng. Chem. Res. 1990, 29, 2284-2294.

864 [10]Gross, J.; Sadowski, G. Perturbed-chain SAFT: An equation of state based on a 865 perturbation theory for chain molecules. Ind. Eng. Chem. Res. 2001, 40, 1244-1260.

866 [11] Gil-Villegas, A.; Galindo, A.; Whitehead, P. J.; Mills, S. J.; Jackson, G.; Burgess, A. 867 N. Statistical associating fluid theory for chain molecules with attractive potentials of 868 variable range. J. Chem. Phys. 1997, 106, 4168-4186.

869 [12] Galindo, A.; Davies, L. A.; Gil-Villegas, A.; Jackson, G. The thermodynamics of 870 mixtures and the corresponding mixing rules in the SAFT-VR approach for potentials 871 of variable range. Mol. Phys. 1998, 93, 241-252.

872 [13]Blas, F. J.; Vega, L. F. Thermodynamic behaviour of homonuclear and heteronuclear 873 Lennard-Jones chains with association sites from simulation and theory. Mol. Phys. $874 \quad 1997,92,135-150$.

875 [14]Chen, J.; Mi, J. G. Equation of state extended from SAFT with improved results for 876 non-polar fluids across the critical point. Fluid Phase Equilib. 2001, 186, 165-184.

877 [15] Polishuk, I. Hybridizing SAFT and cubic EOS: what can be achieved? Ind. Eng. Chem. $878 \quad$ Res. 2011, 50, 4183-4198.

879 [16]Kontogeorgis, G. M.; Voutsas, E. C.; Yakoumis, I. V.; Tassios, D. P. An equation of $880 \quad$ state for associating fluids. Ind. Eng. Chem. Res. 1996, 35, 4310-4318. 
[17] Kontogeorgis, G. M.; Folas, G. K. Thermodynamic Models for Industrial Applications: from Classical and Advanced Mixing Rules to Association Theories. John Wiley \& Sons: Chichester, U.K., 2009.

[18]P Palma, A. M.; Oliveira, M. B.; Queimada, A. J.; Coutinho, J. A. Evaluating cubic plus association equation of state predictive capacities: A study on the transferability of the hydroxyl group associative parameters. Ind. Eng. Chem. Res. 2017, 56, 7086-7099.

[19]Palma, A. M.; Queimada, A. J.; Coutinho, J. A. Improved prediction of water properties and phase equilibria with a modified cubic plus association equation of state. Ind. Eng. Chem. Res. 2017, 56, 15163-15176.

[20]Palma, A. M.; Oliveira, M. B.; Queimada, A. J.; Coutinho, J. A. Re-evaluating the CPA EoS for improving critical points and derivative properties description. Fluid Phase Equilib. 2017, 436, 85-97.

[21]Polishuk, I. Generalization of SAFT + Cubic equation of state for predicting and correlating thermodynamic properties of heavy organic substances. J. Supercrit. Fluids 2012, 67, 94-107.

[22] Polishuk, I.; Perel, A. Implementation of PC-SAFT and SAFT + Cubic for modeling thermodynamic properties of eight 1-alkenes and their mixtures. J. Chem. Thermodyn. 2012, 54, 155-164.

[23]Polishuk, I.; Katz, M.; Levi, Y.; Lubarsky, H. Implementation of PC-SAFT and SAFT + Cubic for modeling thermodynamic properties of haloalkanes. I. 11 halomethanes. 
Fluid Phase Equilib. 2012, 316, 66-73.

903 [24]Polishuk, I.; Assor, E.; Cohen, N.; Potievsky, R. Implementation of PC-SAFT and 904 SAFT + Cubic for modeling thermodynamic properties of haloalkanes. II. 7 905 Haloethanes and their mixtures. Int. J. Refrig. 2013, 36, 980-991.

906 [25] Polishuk, I. Implementation of SAFT + Cubic and PC-SAFT for comprehensive 907 description of thermodynamic properties of n-octane and its mixtures. J. Supercrit. $908 \quad$ Fluids 2012, 62, 47-54.

909 [26] Polishuk, I. Semi-Theoretical Versus Entirely Empirical: Comparing SAFT + Cubic 910 and Soave-Benedict-Webb-Rubin (SBWR) Equations of State. Ind. Eng. Chem. Res. $911 \quad 2011,50,11422-11431$.

912 [27] Polishuk, I. Implementation of perturbed-chain statistical associating fluid theory (PC913 SAFT), generalized (G) SAFT + Cubic, and cubic-plus-association (CPA) for 914 modeling thermophysical properties of selected 1-alkyl-3-methylimidazolium ionic 915 liquids in a wide pressure range. J. Phys. Chem. A 2013, 117, 2223-2232.

916 [28] Polishuk, I. Implementation of CP-PC-SAFT for predicting thermodynamic properties 917 and gas solubility in 1-alkyl-3-methylimidazolium bis (trifluoromethylsulfonyl) imide 918 ionic liquids without fitting binary parameters. Ind. Eng. Chem. Res. 2017, 56, $919 \quad 7845-7857$.

920 [29]Polishuk, I.; Sidik, Y.; NguyenHuynh, D. Predicting phase behavior in aqueous 921 systems without fitting binary parameters I: CP-PC-SAFT EOS, aromatic compounds. $922 \quad$ AIChE J. 2017, 63, 4124-4135. 
923 [30]Lubarsky, H.; Polishuk, I.; NguyenHuynh, D. The group contribution method (GC) 924 versus the critical point-based approach (CP): predicting thermodynamic properties of 925 weakly-and non-associated oxygenated compounds by GC-PPC-SAFT and CP-PC$926 \quad$ SAFT. J. Supercrit. Fluids 2016, 110, 11-21.

927 [31]Liang, X.; Tsivintzelis, I.; Kontogeorgis, G. M. Modeling water containing systems 928 with the simplified PC-SAFT and CPA equations of state. Ind. Eng. Chem. Res. 2014, $929 \quad 53,14493-14507$.

930 [32]Diamantonis, N. I.; Economou, I. G. Evaluation of statistical associating fluid theory 931 (SAFT) and perturbed chain-SAFT equations of state for the calculation of 932 thermodynamic derivative properties of fluids related to carbon capture and 933 sequestration. Energy Fuels 2011, 25, 3334-3343.

934 [33]De Villiers, A. J.; Schwarz, C. E.; Burger, A. J.; Kontogeorgis, G. M. Evaluation of the 935 PC-SAFT, SAFT and CPA equations of state in predicting derivative properties of 936 selected non-polar and hydrogen-bonding compounds. Fluid Phase Equilib. 2013, 338, $937 \quad 1-15$.

938 [34]Burgess, W. A.; Tapriyal, D.; Gamwo, I. K.; Wu, Y.; McHugh, M. A.; Enick, R. M. 939 New group-contribution parameters for the calculation of PC-SAFT parameters for use 940 at pressures to $276 \mathrm{MPa}$ and temperatures to $533 \mathrm{~K}$. Ind. Eng. Chem. Res. 2014, 53, $941 \quad 2520-2528$.

942 [35]Lafitte, T.; Piñeiro, M. M.; Daridon, J. L.; Bessières, D. A comprehensive description 943 of chemical association effects on second derivative properties of alcohols through a 
SAFT-VR approach. J. Phys. Chem. B 2007, 111, 3447-3461.

945 [36]Lafitte, T.; Bessieres, D.; Piñeiro, M. M.; Daridon, J. L. Simultaneous estimation of

946 phase behavior and second-derivative properties using the statistical associating fluid

947 theory with variable range approach. J. Chem. Phys. 2006, 124, 024509.

948 [37]Lafitte, T.; Apostolakou, A.; Avendano, C.; Galindo, A.; Adjiman, C. S.; Müller, E. A.;

949 Jackson, G. Accurate statistical associating fluid theory for chain molecules formed

950 from Mie segments. J. Chem. Phys. 2013, 139, 154504.

951 [38]Perdomo, F. A.; Gil-Villegas, A. Predicting thermophysical properties of biodiesel fuel

952 blends using the SAFT-VR approach. Fluid Phase Equilib. 2011, 306, 124-128.

953 [39] Oliveira, M. B.; Llovell, F.; Coutinho, J. A. P.; Vega, L. F. New procedure for

954 enhancing the transferability of statistical associating fluid theory (SAFT) molecular

955 parameters: the role of derivative properties. Ind. Eng. Chem. Res. 2016, 55,

$956 \quad 10011-10024$.

957 [40]Llovell, F.; Vega, L. F. Prediction of thermodynamic derivative properties of pure 958 fluids through the soft-SAFT equation of state. J. Phys. Chem. B 2006, 110, $959 \quad 11427-11437$.

960 [41]Llovell, F.; Peters, C. J.; Vega, L. F. Second-order thermodynamic derivative properties 961 of selected mixtures by the soft-SAFT equation of state. Fluid Phase Equilib. 2006, $962 \quad 248,115-122$.

963 [42]Dias, A. M. A.; Llovell, F.; Coutinho, J. A. P.; Marrucho, I. M.; Vega, L. F. 964 Thermodynamic characterization of pure perfluoroalkanes, including interfacial and 
second order derivative properties, using the crossover soft-SAFT EoS. Fluid Phase

966

967

968

969

970

971

972

973

974

975

976

977

978

979

980

981

982

983

984

985

Equilib. 2009, 286, 134-143.

[43] Maghari, A.; Sadeghi, M. S. Prediction of sound velocity and heat capacities of nalkanes from the modified SAFT-BACK equation of state. Fluid Phase Equilib. 2007, $252,152-161$.

[44] Maghari, A.; Hamzehloo, M. Second-order thermodynamic derivative properties of binary mixtures of n-alkanes through the SAFT-CP equation of state. Fluid Phase Equilib. 2011, 302, 195-201.

[45] Shen, G.; Held, C.; Lu, X.; Ji, X. Modeling thermodynamic derivative properties of ionic liquids with ePC-SAFT. Fluid Phase Equilib. 2015, 405, 73-82.

[46]Diedrichs, A.; Rarey, J.; Gmehling, J. Prediction of liquid heat capacities by the group contribution equation of state VTPR. Fluid Phase Equilib. 2006, 248, 56-69.

[47]Alavianmehr, M. M.; El-Shaikh, M.; Akbari, F.; Behjatmanesh-Ardakani, R. A new equation of state for modeling thermodynamic properties of some fatty acids alkyl esters, methyl ester-based biodiesels and their blends. Fluid Phase Equilib. 2017, 442, $53-61$

[48]Ceriani, R.; Gani, R.; Meirelles, A. J. Prediction of heat capacities and heats of vaporization of organic liquids by group contribution methods. Fluid Phase Equilib. 2009, 283, 49-55.

[49] Gardas, R. L.; Coutinho, J. A. A group contribution method for heat capacity estimation of ionic liquids. Ind. Eng. Chem. Res. 2008, 47, 5751-5757. 
[50] Soriano, A. N.; Agapito, A. M.; Lagumbay, L. J. L. I.; Caparanga, A. R.; Li, M. H. A simple approach to predict molar heat capacity of ionic liquids using group-additivity method. J. Taiwan Inst. Chem. Eng. 2010, 41, 307-314.

[51] Valderrama, J. O.; Toro, A.; Rojas, R. E. Prediction of the heat capacity of ionic liquids using the mass connectivity index and a group contribution method. J. Chem. Thermodyn. 2011, 43, 1068-1073.

[52]Cedeño, F. O.; Prieto, M. M.; Xiberta, J. Measurements and estimate of heat capacity for some pure fatty acids and their binary and ternary mixtures. J. Chem. Eng. Data 2000, 45, 64-69.

[53] Anand, K.; Sharma, R. P.; Mehta, P. S. A comprehensive approach for estimating thermo-physical properties of biodiesel fuels. Appl. Therm. Eng. 2011, 31, 235-242.

[54]Bolmatov, D.; Brazhkin, V. V.; Trachenko, K. The phonon theory of liquid thermodynamics. Sci. Rep. 2012, 2, 1-6.

[55]Dadgostar, N.; Shaw, J. M. A predictive correlation for the constant-pressure specific heat capacity of pure and ill-defined liquid hydrocarbons. Fluid Phase Equilib. 2012, $313,211-226$

[56]Dadgostar, N.; Shaw, J. M. Predictive correlations for liquid heat capacity-including the critical region. Fluid Phase Equilib. 2013, 344, 139-151.

[57] Jaubert, J. N.; Privat, R.; Le Guennec, Y.; Coniglio, L. Note on the properties altered by application of a Péneloux-type volume translation to an equation of state. Fluid Phase Equilib. 2016, 419, 88-95. 
1007

1008

1009

1010

1011

1012

1013

1014

1015

1016

1017

1018

1019

1020

1021

1022

1023

1024

1025

1026

1027

[58]NIST Chemistry Webbook. http://webbook.nist.gov/chemistry (accessed May 2020).

[59]Poling, B. E.; Prausnitz, J. M.; O’connell, J. P. The Properties of Gases and Liquids, 5th ed; Mcgraw-hill: New York, 2001.

[60]Liang, X.; Maribo-Mogensen, B.; Thomsen, K.; Yan, W.; Kontogeorgis, G. M. Approach to improve speed of sound calculation within PC-SAFT framework. Ind. Eng. Chem. Res. 2012, 51, 14903-14914.

[61]DIPPR 801 database, Design Institute for Physical Property Data. AIChE: New York, 1998.

[62]Polishuk, I. Till which pressures the fluid phase EOS models might stay reliable? J. Supercrit. Fluids 2011, 58, 204-215.

[63] Polishuk, I. Addressing the issue of numerical pitfalls characteristic for SAFT EOS models. Fluid Phase Equilib. 2011, 301, 123-129.

[64]Polishuk, I.; Wisniak, J.; Segura, H. Some observations regarding the prediction of isochoric heat capacities by engineering EOS models. Fluid Phase Equilib. 2009, 277, 121-125.

[65] Miyazawa, T.; Kondo, S.; Suzuki, T.; Sato, H. Specific heat capacity at constant pressure of ethanol by flow calorimetry. J. Chem. Eng. Data 2012, 57, 1700-1707.

[66]Zhu, C.; Yang, F.; Liu, X.; He, M. Isobaric molar heat capacities measurement of binary mixtures containing ethyl laurate and ethanol at high pressures. J. Mol. Liq. 2019, 280, 301-306.

[67] Vega-Maza, D.; Segovia, J. J.; Martín, M. C.; Villamañán, R. M.; Villamañán, M. A. 
Thermodynamic properties of biofuels: Heat capacities of binary mixtures containing ethanol and hydrocarbons up to $20 \mathrm{MPa}$ and the pure compounds using a new flow calorimeter. J. Chem. Thermodyn. 2011, 43, 1893-1896.

[68] Stromsoe, E.; Ronne, H. G.; Lydersen, A. L. Heat capacity of alcohol vapors at atmospheric pressure. J. Chem. Eng. Data 1970, 15, 286-290.

[69]Counsell, J. F.; Lees, E. B.; Martin, J. F. Thermodynamic properties of organic oxygen compounds. Part XIX. Low-temperature heat capacity and entropy of propan-1-ol, 2methylpropan-1-ol, and pentan-1-ol. J. Chem. Soc. A 1968, 1819-1823.

[71]Torín-Ollarves, G. A.; Segovia, J. J.; Martín, M. C.; Villamañán, M. A.

[72] Naziev, Y. M.; Bashirov, M. M.; Badalov, Y. A. Experimental device for measurement of isobaric specific heat of electrolytes at high state parameters. J. Eng. Phys. 1986,

1048 [74]Domańska, U.; Marciniak, M. Experimental solid-liquid equilibria for systems 
containing alkan-1-ol + 1, 3-diaminopropane: Heat capacities of alkan-1-ols and amines - Thermodynamic functions of dissociation and enthalpies of melting of the congruently melting compounds for the systems (alkan-1-ol + amine). Fluid Phase Equilib. 2005, 235, 30-41.

[75]Casás, L. M.; Plantier, F.; Piñeiro, M. M.; Legido, J. L.; Bessières, D. Calibration of a low temperature calorimeter and application in the determination of isobaric heat capacity of 2-propanol. Thermochim. Acta 2010, 507, 123-126. Chem. Thermodyn. 2017, 111, 41-51.

[80]Dzida, M.; Góralski, P. Molar heat capacities for (2-methyl-2-butanol + heptane) 
2009, 41, 402-413. 
1071 Abstract Graphics ${ }^{\text {a }}$

1072
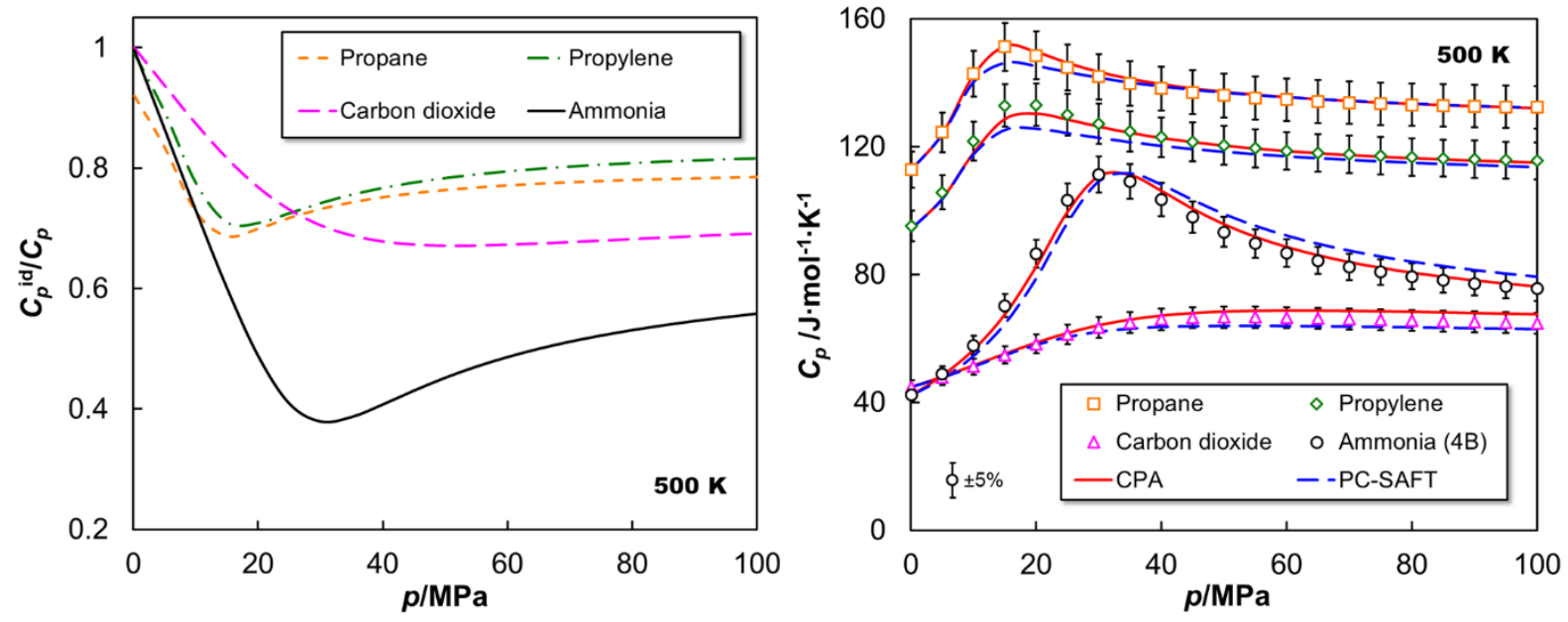

$1073{ }^{a}$ For Table of Contents use only; 